\title{
Dnmt3b ablation impairs fracture repair through upregulation of Notch pathway
}

\author{
Jun Ying, ${ }^{1,2,3}$ Taotao Xu, ${ }^{1,2,3}$ Cuicui Wang, ${ }^{1}$ Hongting Jin, ${ }^{2,3}$ Peijian Tong, ${ }^{2}$ Jianjun Guan, ${ }^{4}$ \\ Yousef Abu-Amer, ${ }^{1,5}$ Regis O'Keefe, ${ }^{1}$ and Jie Shen ${ }^{1}$ \\ 'Department of Orthopaedic Surgery, School of Medicine, Washington University in St. Louis, St. Louis, Missouri, USA. \\ ${ }^{2}$ Institute of Orthopaedics and Traumatology, First Affiliated Hospital of Zhejiang Chinese Medical University, \\ Hangzhou, China. ${ }^{3}$ Zhejiang Chinese Medical University, Hangzhou, China. ${ }^{4}$ Department of Biomedical Engineering, \\ School of Engineering, Washington University in St. Louis, St. Louis, Missouri, USA. ${ }^{5}$ Shriners Hospital for Children, \\ St. Louis, Missouri, USA.
}

\begin{abstract}
We previously established that DNA methyltransferase $3 b$ (Dnmt3b) is the sole Dnmt responsive to fracture repair and that Dnmt3b expression is induced in progenitor cells during fracture repair. In the current study, we confirmed that Dnmt3b ablation in mesenchymal progenitor cells (MPCs) resulted in impaired endochondral ossification, delayed fracture repair, and reduced mechanical strength of the newly formed bone in Prx1-Cre;Dnmt3bf/f $\left(D n m t 3 b^{P r x}\right)$ mice. Mechanistically, deletion of Dnmt3b in MPCs led to reduced chondrogenic and osteogenic differentiation in vitro. We further identified Rbpjк as a downstream target of Dnmt3b in MPCs. In fact, we located 2 Dnmt3b binding sites in the murine proximal Rbpjк promoter and gene body and confirmed Dnmt3b interaction with the 2 binding sites by ChIP assays. Luciferase assays showed functional utilization of the Dnmt3b binding sites in murine C3H10T1/2 cells. Importantly, we showed that the MPC differentiation defect observed in Dnmt3b deficiency cells was due to the upregulation of Rbpjк, evident by restored MPC differentiation upon Rbpjк inhibition. Consistent with in vitro findings, Rbpjк blockage via dual antiplatelet therapy reversed the differentiation defect and accelerated fracture repair in Dnmt3b ${ }^{\text {Prx }}$ mice. Collectively, our data suggest that Dnmt3b suppresses Notch signaling during MPC differentiation and is necessary for normal fracture repair.
\end{abstract}

Authorship note: JY and TX contributed equally to this work.

Conflict of interest: The authors have declared that no conflict of interest exists.

Copyright: (c) 2020, American Society for Clinical Investigation.

Submitted: July 18, 2019 Accepted: December 26, 2019 Published: February 13, 2020.

Reference information: JCI Insight. 2020;5(3):e131816.

https://doi.org/10.1172/jci. insight.131816.

\section{Introduction}

Bone fractures commonly result in prolonged disability and increased socioeconomic burden (1). In the United States, approximately 1.6 million bone fractures had impaired healing or progress to nonunion each year, further compromising the quality of life and life expectancy of many patients (2-4). Bone fracture injuries disrupt marrow sinusoidal architecture and surrounding soft tissues, and blood clots form in the fracture area that initiate an early local inflammatory process (5). During this early hematoma period, endogenous mesenchymal progenitor cells (MPCs), recruited from multiple sources, undergo precisely regulated multidirectional differentiation to generate the cell lineages involved in bone regeneration and vascularization (6-8). In particular, MPC-derived chondrocytes and osteoblasts are essential cell populations necessary for cartilaginous and bony callus formation. Differentiation of these cells along their lineages results in fracture union and return of the mechanical integrity of the skeleton $(9,10)$. After remodeling by osteoclasts, the fractured bone returns to its original cellular composition, morphologic structure, and biomechanical function (1). Fracture repair integrates cytokines, growth factors, and signaling pathways as well as progenitor cell proliferation and differentiation in a highly regulated process that leads to tissue regeneration $(7,11,12)$. Although extensive studies define the cellular and molecular contributions to fracture repair, the effect of epigenetic regulations in fracture repair, particularly MPC differentiation, is still largely unknown.

In mammals, there are 3 epigenetic regulatory processes: DNA methylation, histone modification, and noncoding RNAs (13). Recent epigenome studies of fracture patients revealed differential methylation loci associated with stem cell proliferation and differentiation in human mesenchymal stem cells, suggesting that DNA methylation is involved in fracture repair processes (14). DNA methylation is a well-characterized epigenetic modification in CpG-rich islands, leading to gene activation or suppression dependent on methylation patterns of the promoter, enhancer, and gene body $(15,16)$. Three independent DNA meth- 
yltransferase (Dnmt) enzymes catalyze the transfer of the methyl groups from S-adenosyl-L-methionine to cytosine in mammals. Dnmt1 is largely responsible for the maintenance of genome-wide methylation during cell proliferation, whereas Dnmt3a and Dnmt3b primarily act as de novo Dnmts to establish unique DNA methylation patterns during development (13). The biological functions of the de novo Dnmts in the regulation of postnatal tissue repair processes are areas of active investigation. Recent studies defined essential roles of Dnmt3a and Dnmt3b in adult stem cell and hematopoietic stem cell differentiation (17, 18) and in osteoclastogenesis (19). Moreover, our recent findings provided evidence that Dnmt3b is highly expressed in chondrocytes and progenitor cells of fracture calluses and showed that Dnmt3b ablation in chondrocytes results in a chondrogenic differentiation defect and delayed fracture repair (20). This suggests that Dnmt3b has an essential role in regulation of progenitor cell differentiation.

Therefore, this study explores the mechanism involved in the regulation of MPC proliferation and differentiation by Dnmt3b in both chondrogenic and osteogenic lineages during bone fracture healing. We show that ablation of Dnmt3b is associated with reduced chondrogenic and osteogenic differentiation in MPCs, leading to decreased cartilaginous and bony callus formation and impaired fracture repair in mice that is due

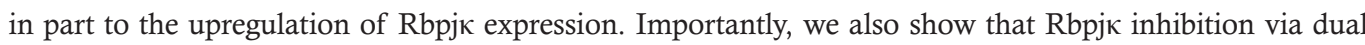
antiplatelet therapy (DAPT) restores the Dnmt3b loss-of-function-induced (LOF-induced) MPC differentiation defect in cell cultures and fracture healing delay in mice. To our knowledge these findings define new processes, pathways, and therapeutic targets to enhance fracture repair and restore skeletal mobility.

\section{Results}

Ablation of Dnmt3b impairs endochondral ossification in fracture repair. We previously identified Dnmt3b as the most regulated epigenetic factor responsive to fracture repair process. Dnmt3b expression is induced and maintained in MPCs during fracture repair and gradually declined to a nondetectable level (20). Importantly, in the current study, we revealed a reduction of Dnmt3b expression in fracture calluses 10 days after fracture (dpf) in high-fat diet-induced (HFD-induced) diabetic mice and aging mice (Supplemental Figure 1, A and B; supplemental material available online with this article; https://doi.org/10.1172/jci.insight.131816DS1), suggesting an essential role of Dnmt3b in the regulation of fracture repair. To comprehensively examine

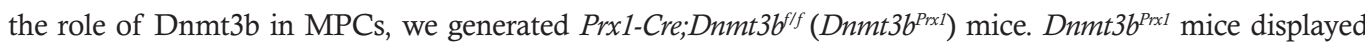
no obvious growth difference, with similar body weight and size (Supplemental Figure 2A), compared with Cre-negative littermates through 10 weeks of age. Microcomputed tomography (micro-CT) analysis demonstrated similar cortical thickness, tissue mineral density, trabecular thickness, and bone volume/total volume in both $D n m t 3 b^{P r x}$ mice and littermate controls (Supplemental Figure 2, B-D), confirming our observations regarding relative normal bone development in $D n m t 3 b^{P r x}$ mice. This suggests that loss of Dnmt3b is either not critical or is compensated by the presence of other Dnmts during bone development. We next assessed the Prx1-Cre-mediated Dnmt3b deletion efficiency in the tibiae of adult Dnmt $3 b^{P r x 1}$ mice and in fracture callus tissues. Western blot revealed substantial Dnmt3b reduction in protein lysates extracted from intact tibiae of 10-week-old Dnmt3 $b^{P x I}$ mice (Supplemental Figure 3A), indicating efficient Dnmt3b deletion in MPCs and their derived osteoblasts. As expected, IHC analyses of fracture calluses $10 \mathrm{dpf}$ in control mice and Dnmt3b $b^{\text {Prx }}$ mice also confirmed an efficient deletion of Dnmt3b, specifically in MPCs (Supplemental Figure 3B).

We then performed tibia fracture on 10-week-old Dnmt $3 b^{P r x 1}$ mice and their littermate controls to determine the effect of Dnmt3b deletion on MPC proliferation and differentiation as well as on fracture repair in mice. Alcian blue/Hematoxylin/Orange G (ABH/OG) staining showed impaired fracture repair in Dnmt3b${ }^{P r x l}$ mice. By $7 \mathrm{dpf}$, control mice had abundant chondrogenesis at the central hypoxic region of the fracture, and robust osteogenesis and intramembranous bone formation in the periosteum adjacent to the fractures site. In contrast, fractures in $D n m t 3 b^{P r x l}$ mice were composed primarily of undifferentiated mesenchyme tissue with limited chondrocyte cartilage or periosteal bone formation. Fractures in control mice had limited mesenchyme with the presence of mature cartilage tissues and abundant bone formation $10 \mathrm{dpf}$, and the cartilage template was largely resorbed and replaced by secondary bone formation $14 \mathrm{dpf}$ (Figure 1A). In comparison, mesenchymal tissue remained in fracture calluses $14 \mathrm{dpf}$ in $D n m t 3 b^{P x x}$ mice. Quantitative histomorphometry confirmed that there was less mesenchymal tissue in fracture calluses of $D n m t 3 b^{P r x I}$ mice during the early stages of fracture healing and that $D n m t 3 b^{P_{x x l}}$ mice exhibited a continued presence of mesenchymal tissues and reduced cartilage and intramembranous bone formation through $14 \mathrm{dpf}$ compared with control mice (Figure 1B). Micro-CT analyses of mineralized calluses in control mice showed robust bone formation surrounding the fracture site 10 and $14 \mathrm{dpf}$. In contrast, the fracture healing was significantly delayed in $D n m t 3 b^{P_{r x I}}$ mice, as 
A

A
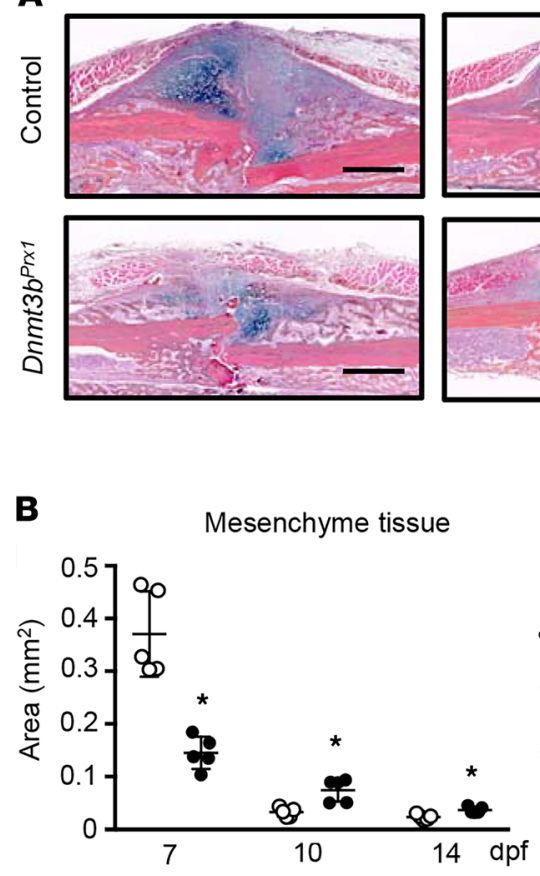

C
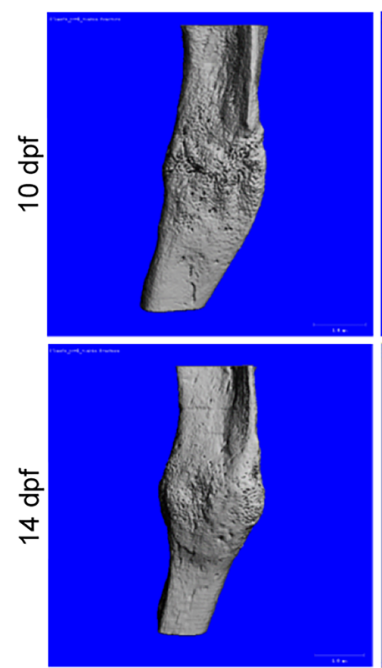

$10 \mathrm{dpf}$
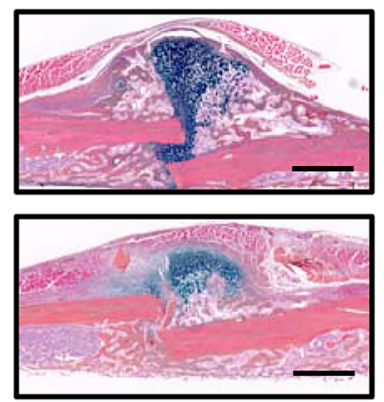

$14 \mathrm{dpf}$
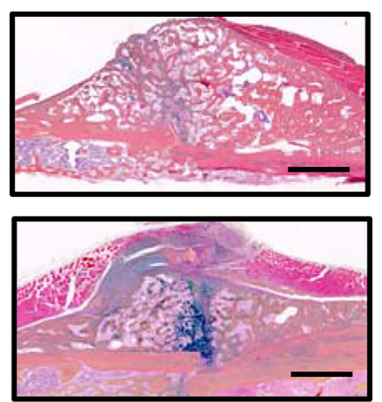

O Control

- Dnmt3bPrx1
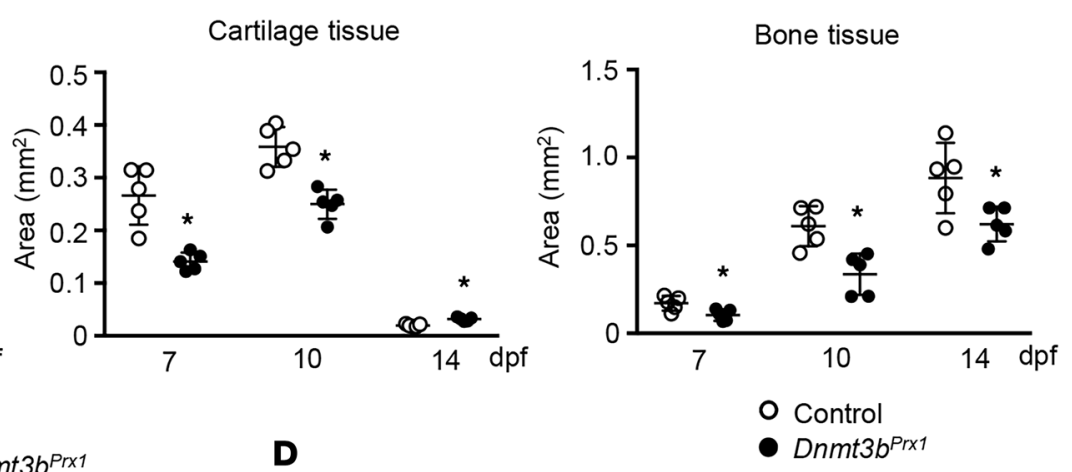

D
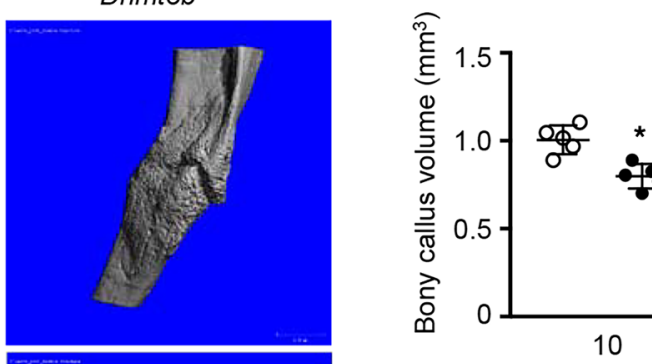

- Dnmt3b

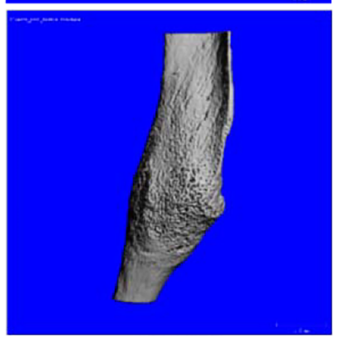

10

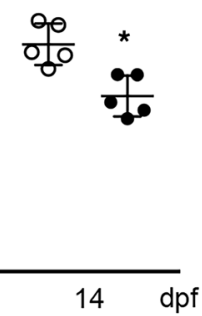

Figure 1. Ablation of Dnmt3b in mesenchymal progenitor cells reduced cartilaginous callus formation and bony callus ossification during fracture repair. (A) Alcian blue/Hematoxylin/Orange G (ABH/OG) staining of fracture callus sections of Dnmt3 $b^{\text {Prx }}$ mice and control mice at the indicated times ( $n$ = 5). Scale bar: $500 \mu \mathrm{m}$. (B) Histomorphometry quantification of the mesenchyme, cartilage, and bone areas was performed on ABH/OG-stained fracture callus sections of $D n m t 3 b^{P r x}$ mice and control mice at the indicated times $(n=5)$. (C) Microcomputed tomography assessment of mineralized bone in fracture calluses of $D n m t 3 b^{P_{r \times 1}}$ mice and control mice at the indicated times $(n=5)$. (D) Quantification of bony callus volumes in $D n m t 3 b^{P \times x}$ mice and control mice 10 and 14 days after fracture (dpf). Data are presented as mean \pm SD. ${ }^{*} P<0.05$ by 2 -way ANOVA test.

shown by micro-CT, with reduced bony callus 10 and $14 \mathrm{dpf}$ (Figure 1, C and D). Taken together, our in vivo findings revealed a reduced formation of both cartilage and woven bone in the fractures of $D n m t 3 b^{P x I}$ mice, suggesting that the process of chondrogenic and osteogenic differentiation from mesenchymal progenitors is impaired in the absence of Dnmt3b in MPCs during fracture repair.

Ablation of Dnmt3b in vivo leads to delayed bone remodeling and poor healing. Since fracture repair is a highly integrated process, we next examined the effect of loss of Dnmt3b in MPCs on the later stages of healing. Tartrate-resistant acid phosphatase (TRAP) staining was performed to examine osteoclast-mediated remodeling 14,21 , and $28 \mathrm{dpf}$. Consistent with our previous finding and others' reports $(1,20-22)$, the total number of 
A
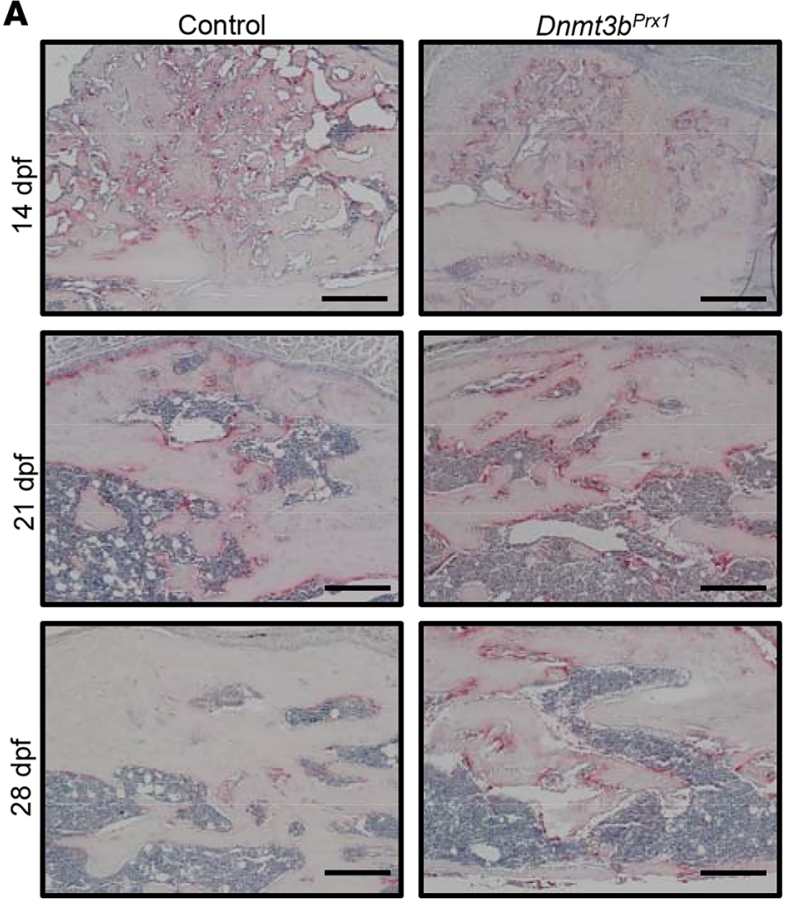

\section{C}

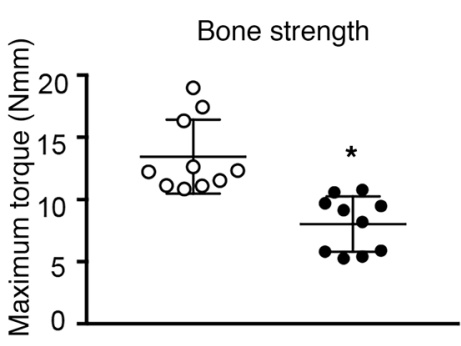

B

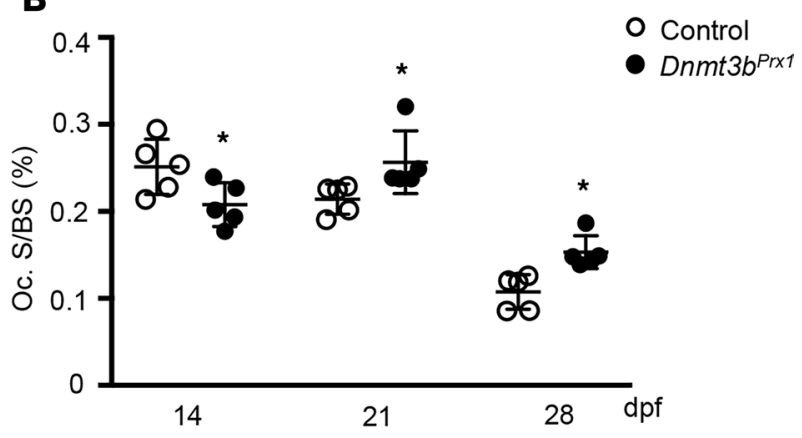

O Control

- Dnmt3bPrx1
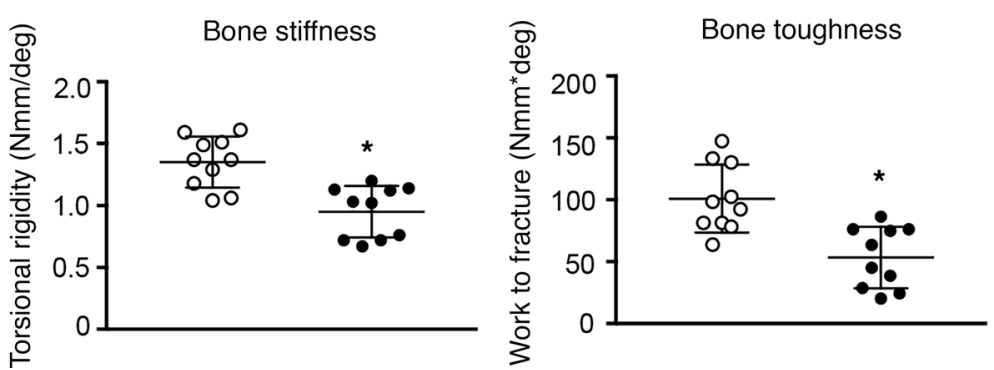

Figure 2. Ablation of Dnmt3b in mesenchymal progenitor cells led to delayed bone callus remodeling and decreased biomechanical properties of the newly formed bone during fracture repair. (A) Tartrate-resistant acid phosphatase (TRAP) staining of fracture callus sections of $D n m t 3 b^{P \times x}$ mice and control mice at the indicated times $(n=5)$. Scale bar: $200 \mu \mathrm{m}$. (B) Histomorphometry analysis of osteoclast surface per bone surface (Oc. S/BS) was performed on TRAP staining of $D n m t 3 b^{P r x}$ and control fractures at the indicated times $(n=5)$. (C) Biomechanical torsion testing was performed on fractured tibiae of $D n m t 3 b^{\text {Prx }}$ mice and control mice 28 days after fracture (dpf). Maximum torque, torsional rigidity, and energy to maximum were measured and calculated to represent the bone strength, bone stiffness, and bone toughness $(n=10)$. Data are presented as mean \pm SD. ${ }^{*} P<0.05$ by 2-way ANOVA test (B) and Student's $t$ test $(\mathbf{C})$.

TRAP-positive cells peaked $14 \mathrm{dpf}$ in the calluses of control mice and gradually decreased to an undetectable level 28 dpf. However, fractures of $D n m t 3 b^{P x l}$ mice displayed a disparate pattern of osteoclast. TRAP-positive cell staining peaked $21 \mathrm{dpf}$ and remained abundant in the calluses of $D n m t 3 b^{P_{x x l}}$ mice throughout the $28 \mathrm{dpf}$ (Figure 2A), leading to increased osteoclast surface per bone surface 21 and $28 \mathrm{dpf}$ (Figure 2B). More importantly, torsion testing performed on fractured tibiae of $D n m t 3 b^{P x l}$ mice and control mice $28 \mathrm{dpf}$ showed that despite comparable bone callus volume $28 \mathrm{dpf}$, the $D n m t 3 b^{P x I}$ mutant fractures had significantly lower mechanical properties, including bone strength (33\% reduction), bone stiffness ( $25 \%$ reduction), and bone toughness ( $50 \%$ reduction) compared with control mice (Figure 2C). Collectively, these in vivo data show that Dnmt3b deficiency in MPCs not only impairs the formation of cartilaginous and bony callus, but also affects later events, including bone remodeling by osteoclasts and the restoration of biomechanical strength.

Dnmt3b ablation reduces MPC differentiation through upregulation of Rbpjk-mediated Notch pathway. To understand the molecular events related to the delay in fracture healing calluses of $D_{n m t}{ }^{P^{P x I}}$ mice, we examined chondrogenic differentiation using limb bud-derived MPCs isolated from E11.5 Dnmt $3 b^{P_{x x l}}$ embryos and control embryos, and osteogenic differentiation on bone marrow-derived MPCs isolated from 10-week-old $D n m t 3 b^{P \times x}$ mice and their littermate controls. Real-time qPCR results confirmed the efficient deletion of Dnmt3b in MPCs from either limb bud or bone marrow (Figure 3, C and F). Of note, Dnmt3b 
deletion did not affect the colony-forming ability from bone marrow-adherent stromal cells (Supplemental 4A). Similar numbers of type I colony-forming-unit fibroblasts, about 60 per $1.5 \times 10^{6}$ marrow cells, were identified in the bone marrow of $D n m t 3 b^{P x x I}$ mice and control mice (Supplemental Figure 4B), suggesting that the population of MPCs and its proliferation capacity were maintained in $D n m t 3 b^{\text {Prxl }}$ mice. We next examined the MPC differentiation potential in vitro and found that following exposure to chondrogenic culture medium for 3 days, MPCs from control limb buds differentiated into chondrocytes and deposited a large amount of cartilage matrix, as reflected by intense Alcian blue staining on the micromass cultures. However, ABH staining was substantially reduced in limb bud-derived MPCs from mice with Dnmt3b deficiency (Figure 3, A and B). In addition, the gene expression studies showed that the chondrogenesis master gene, Sox9, was decreased in limb bud-derived MPCs of Dnmt3b $b^{\text {Prx }}$ mice, as were the other key chondrocyte markers, Col2al and Agcl (Figure 3C), indicating diminished chondrogenic differentiation capacity in MPCs of Dnmt $3 b^{\text {Prxt }}$ mice.

In an analogous manner, bone marrow-derived MPCs from $D n m t 3 b^{P x x}$ mice had reduced osteogenic differentiation (alkaline phosphatase [ALP] staining) and bone mineral deposition (Alizarin red staining) after 7 and 21 days of osteogenic differentiation compared with bone marrow-derived MPCs from control mice (Figure 3, D and E). Moreover, the osteogenic master gene, $S p 7$, as well as Runx2 and Alp were decreased in bone marrow-derived MPCs of $D n m t 3 b^{P x x}$ mice compared with bone marrow-derived MPCs of control mice (Figure 3F). Altogether, deletion of Dnmt3b in vitro led to reduced MPC chondrogenic and osteogenic differentiation, but the cell proliferation was largely unaffected.

The Notch signaling pathway is a key regulator of mesenchymal stem cells differentiation. Active Notch signaling maintains MPCs in a progenitor state and inhibits differentiation, including chondrogenesis and osteogenesis $(3,23,24)$. To determine if Notch signaling is a target of Dnmt3b, we isolated primary MPCs from E11.5 limb bud (Figure 4, A and B) and bone marrow (Figure 4, C and D) and performed real-time qPCR and Western blot to examine the RNA and protein levels of Notch pathway-related genes. In limb budderived MPCs, real-time qPCR analyses confirmed that Dnmt3b deficiency induced the expression of Hey 1 and HeyL (Figure 4A), the critical downstream targets of Notch pathway. Moreover, Western blot revealed that the expression of Rbpjк, a key modulator of Notch, was upregulated in MPCs of Dnmt $3 b^{P r x l}$ mice (Figure 4B). Consistent with the findings in limb bud-derived MPCs, the Rbpjк-mediated Notch pathway was also increased following loss of Dnmt3b in bone marrow-derived MPCs, as was evident by the upregulation of Hey1, HeyL, and Rbpjк in mRNA and protein levels in bone marrow-derived MPCs of Dnmt $3 b^{\text {Prxl }}$ mice (Figure 4, C and D). Altogether, these data indicate that Dnmt3b LOF induces defects of chondrogenic and osteogenic differentiation and results in the upregulation of Rbpjк-mediated Notch pathway in cells.

Mechanistically, with respect to Dnmt3b and Rbpjк, we identified 2 Dnmt3b binding sites (CpG islands 1 and 2, indicated by the green box in Figure 5A) in the murine proximal Rbpjk promoter and gene body regions (which is highly conserved across species; data not shown). Dnmt3b interaction with the 2 binding sites was also shown by ChIP assays (Figure 5B), and Dnmt3b LOF indeed resulted in DNA methylation decreases of $19.25 \%$ (85.46\% in control and $69.02 \%$ in Dnmt3b LOF) and 9.29\% (74.74\% in control and 67.78\% in Dnmt3b LOF) in CpG islands 1 and 2, respectively (Supplemental Figure 5). Luciferase reporter assays further showed functional utilization of the Dnmt $3 \mathrm{~b}$ binding sites in the murine progenitor line, C3H10T1/2 cells (Figure 5C). Dnmt3b LOF increased Rbpjк promoter activity, whereas

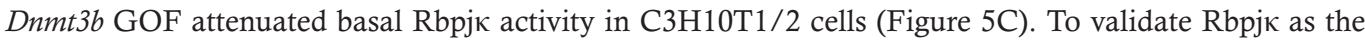
critical downstream target of Dnmt3b, C3H10T1/2 cells were infected with Rbpjк lenti-shRNA in combination with Dnmt3b LOF. Rbpjк inhibition protected MPC differentiation potential by restoring Sox 9 and $S p 7$ expression levels in vitro (Figure 5D), demonstrating that Rbpjк inhibition is able to reverse the chondrogenic and osteogenic differentiation defect mediated by Dnmt3b LOF. Indeed, in the Dnmt $3 b$ and Rbpjк double LOF groups, both chondrocyte matrix deposition and osteoblast differentiation, assessed by $\mathrm{ABH}$ staining and ALP staining, respectively, were restored compared with the Dnmt3b LOF group and even maintained the same enhanced levels as Rbpjк LOF (Figure 5, E and F). Consistently, gene expression levels of chondrocyte matrix markers, $\mathrm{Agcl}$ and Col2, as well as osteoblast differentiation markers, Alp and Spp1, were restored in C3H10T1/2 cells by inhibition of Rbpjк in C3H10T1/2 cells with Dnmt3b LOF (Figure 5G). Together with our in vivo finding that Dnmt3b LOF suppressed MPC differentiation and resulted in impaired fracture healing in mice, these data suggest a mechanism whereby the loss of

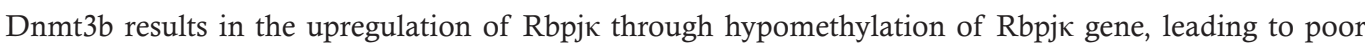
MPC differentiation and bone healing. 
A

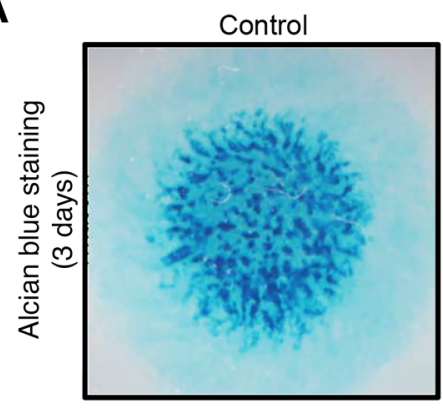

\section{C}
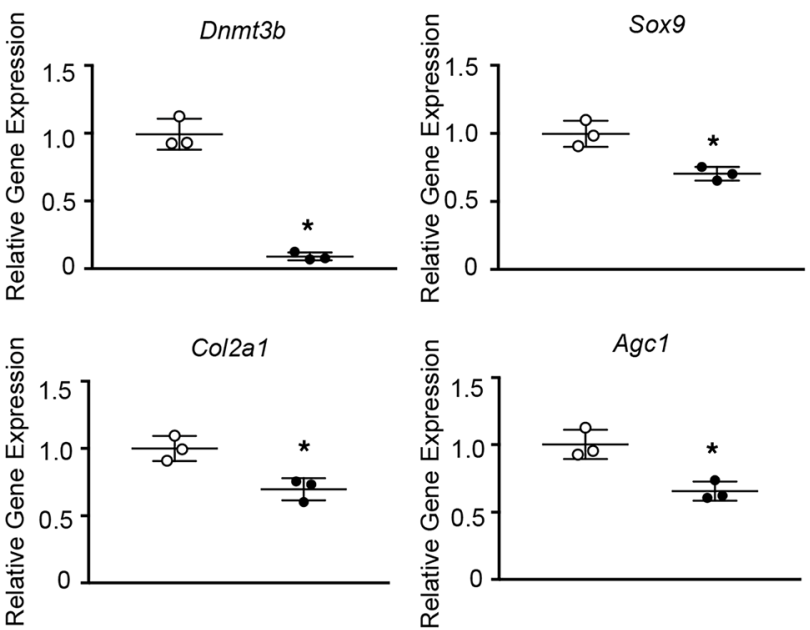

\section{E}
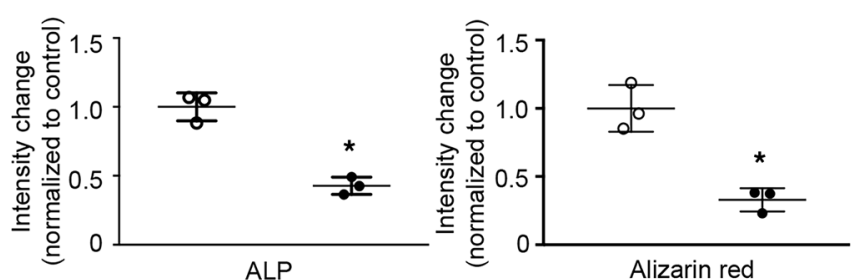

B
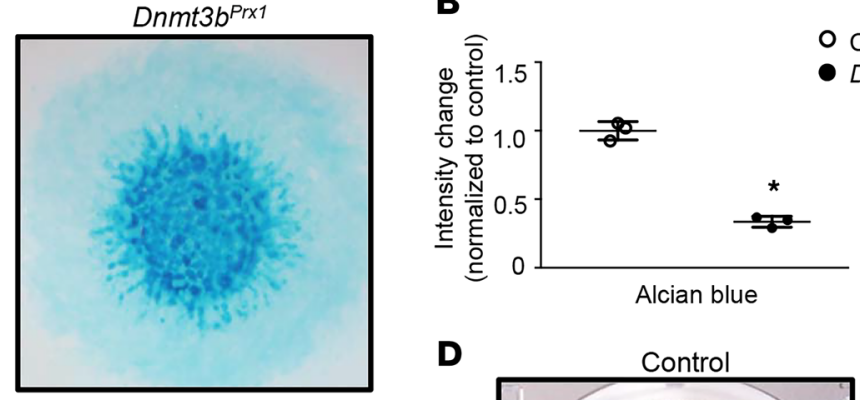

D
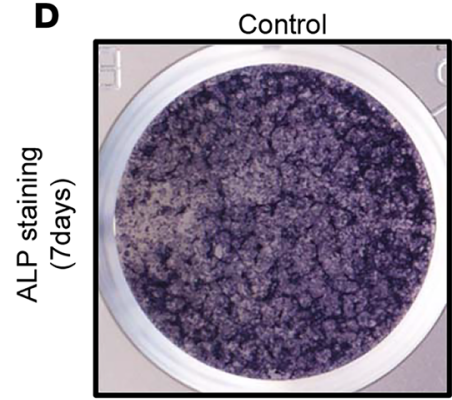

Dnmt3bPrxt
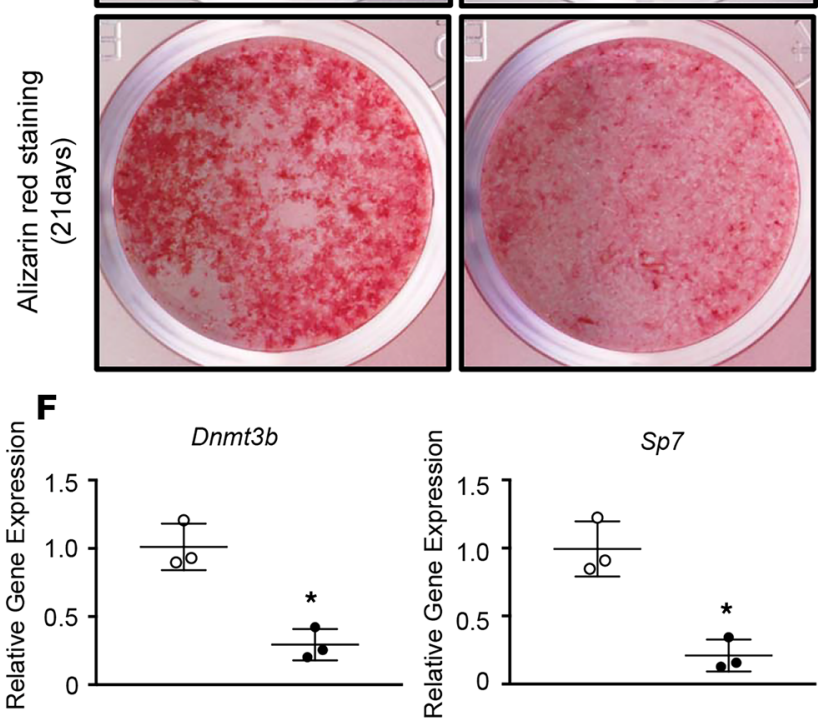

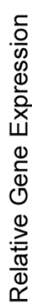

Runx2

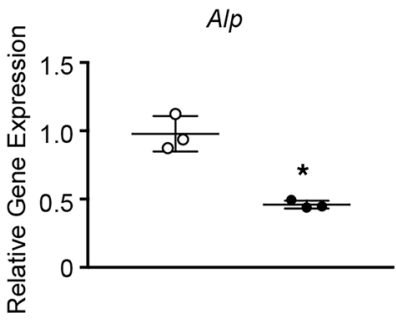

Figure 3. Dnmt3b deletion suppressed mesenchymal progenitor cell chondrogenic and osteogenic differentiation in vitro. Mesenchymal progenitor cell (MPC) were isolated from E11.5 limb bud of Dnmt3b ${ }^{P \times x}$ embryos and control embryos, followed with 3 days micromass culture in the maturation medium. (A) Alcian blue staining was performed on micromass cultures $(n=3)$. (B) Alcian blue staining intensity was measured using NIH Image) software $(n=3)$. (C) Real-time qPCR analyses were performed to determine the relative expression of Dnmt3b, Sox9, Col10a1, and Agc1 in the micromass. The mRNA levels were normalized to that of $A c t b$ and then were normalized to the control group $(n=3)$. (D) Bone marrow-derived MP[s were isolated from 10 -week-old $D n m t 3 b^{P[x]}$ mice and control mice and cultured in osteogenic medium for 4,7 , and 21 days. Alkaline phosphatase (ALP) and Alizarin red stainings were performed on cell cultures at the indicated times $(n=3)$. (E) ALP and Alizarin red intensity was measured using NIH Image) software $(n=3)$. (F) Real-time qPCR analyses were performed to determine the relative expression of $D n m t 3 b, S p 7, R u n \times 2$, and $A / p$ in bone marrow-derived MPCs after 4 days osteogenic culture. The mRNA levels were normalized to that of Actb and then were normalized to the control group $(n=3)$. Data are presented as mean \pm SD. ${ }^{*} P<0.05$ by Student's $t$ test.

Notch inhibition restores the decreased differentiation and fracture repair in Dnmt3 $b^{P_{x x I}}$ mice. It is well known that Rbpjк-mediated Notch pathway is a negative regulator during MPC chondrogenic and osteogenic differentiation $(23,25-27)$, and we previously demonstrated that transient Notch inhibition 
A

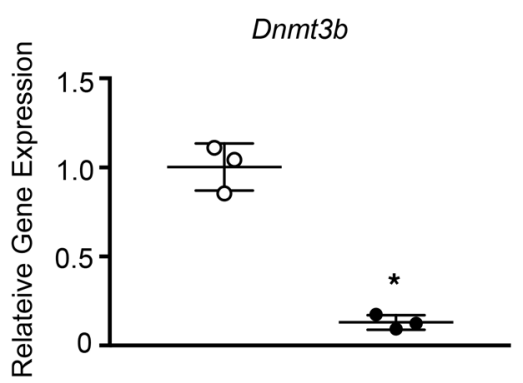

HeyL
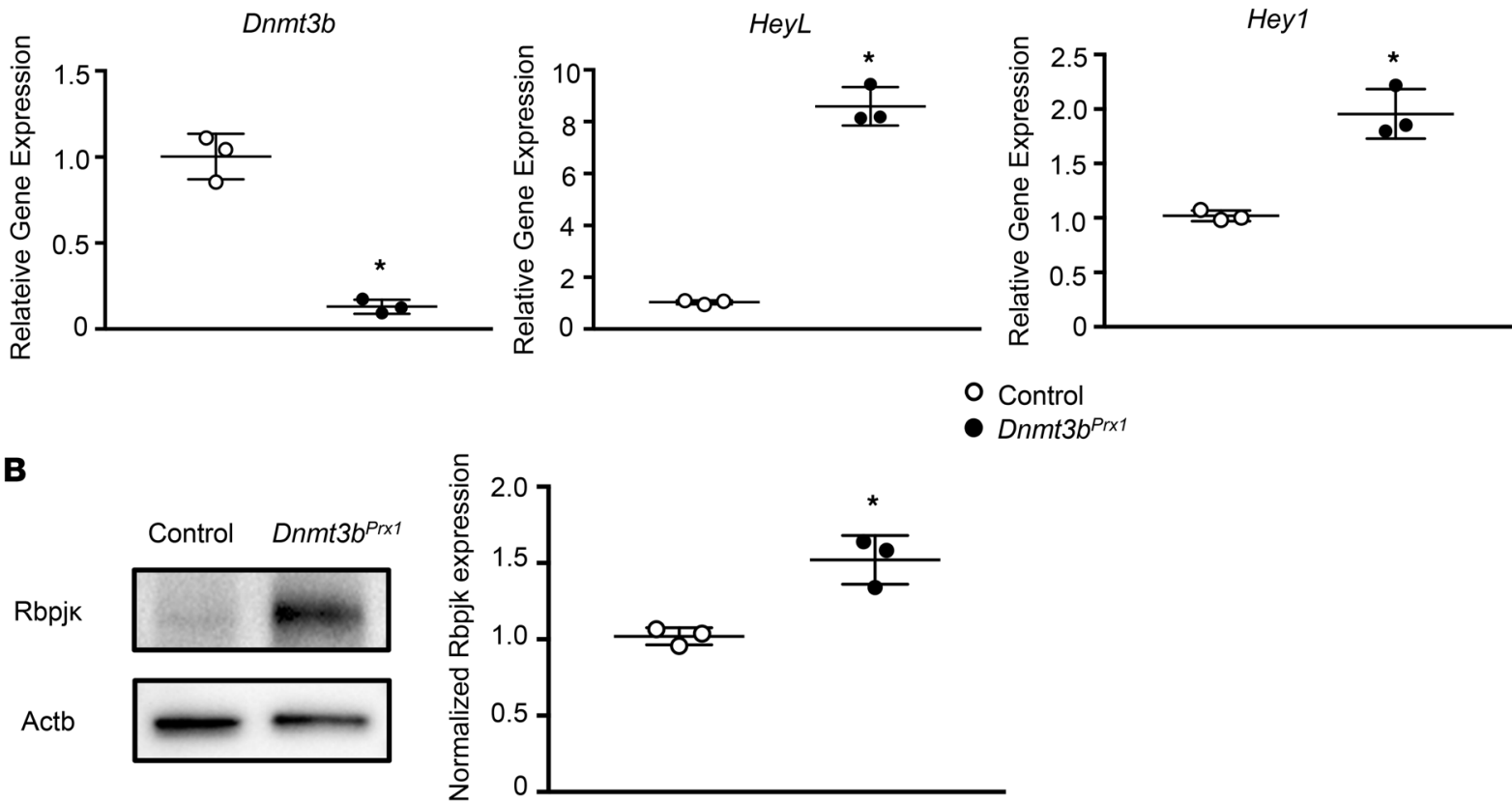

O Control
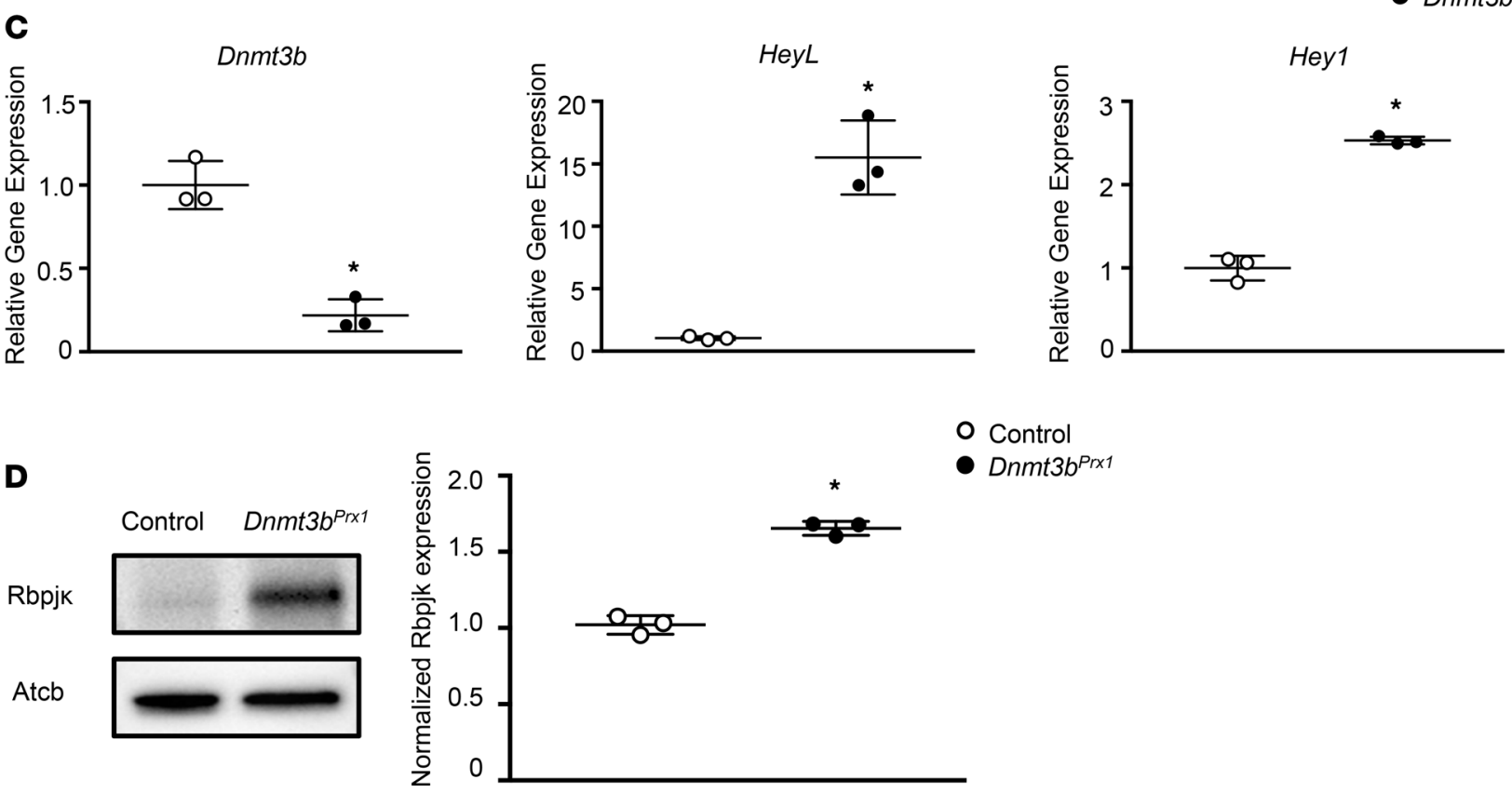

O Control

- Dnmt3b Prxi

Figure 4. Dnmt3b regulated mesenchymal progenitor cell differentiation via Rbpjк-dependent Notch pathway. (A) Real-time qPCR analyses were performed to determine the relative expression of Dnmt3b, Sox9, HeyL, and Hey1 in mesenchymal progenitor cells (MPCs) isolated from E11.5 limb bud of $D n m t 3 b^{P_{r x}}$ embryos and control embryos. The mRNA levels were normalized to that of Actb and then were normalized to the control group $(n=3)$. (B) Western blot was performed to examine the protein level of Rbpjк in MPCs from Dnmt3b $b^{\text {Prx }}$ embryos and control embryos. Quantification of the protein expressions was obtained using NIH ImageJ software $(n=3)$ (C) Real-time qPCR analyses were performed to determine the relative expression of Dnmt3b, HeyL, and Hey1 in bone marrow-derived MPCs from 10-week-old Dnmt3b $b^{P_{r x}}$ mice and control mice. The mRNA levels were normalized to that of Actb and then were normalized to the control group $(n=3)$. (D) Western blot was performed to examine the protein level of Rbpj $\kappa$ in bone marrow-derived MPCs. Quantifications of the protein expressions were obtained using NIH ImageJ software. Data are presented as mean $\pm \mathrm{SD} .{ }^{*} P<0.05$ by Student's $t$ test. 


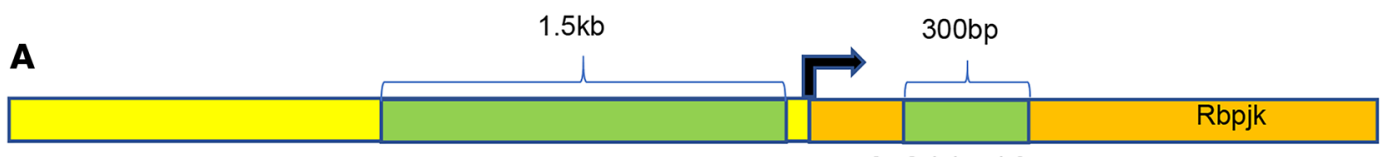

CpG Island 1
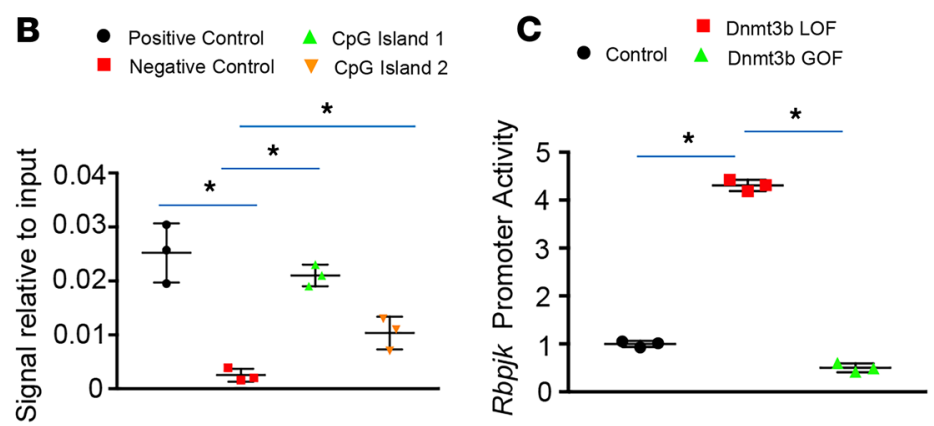

Control $\triangle$ Rbpjk LOF

- Dnmt3b LOF $\nabla$ Dnmt3b; Rbpjk LOF
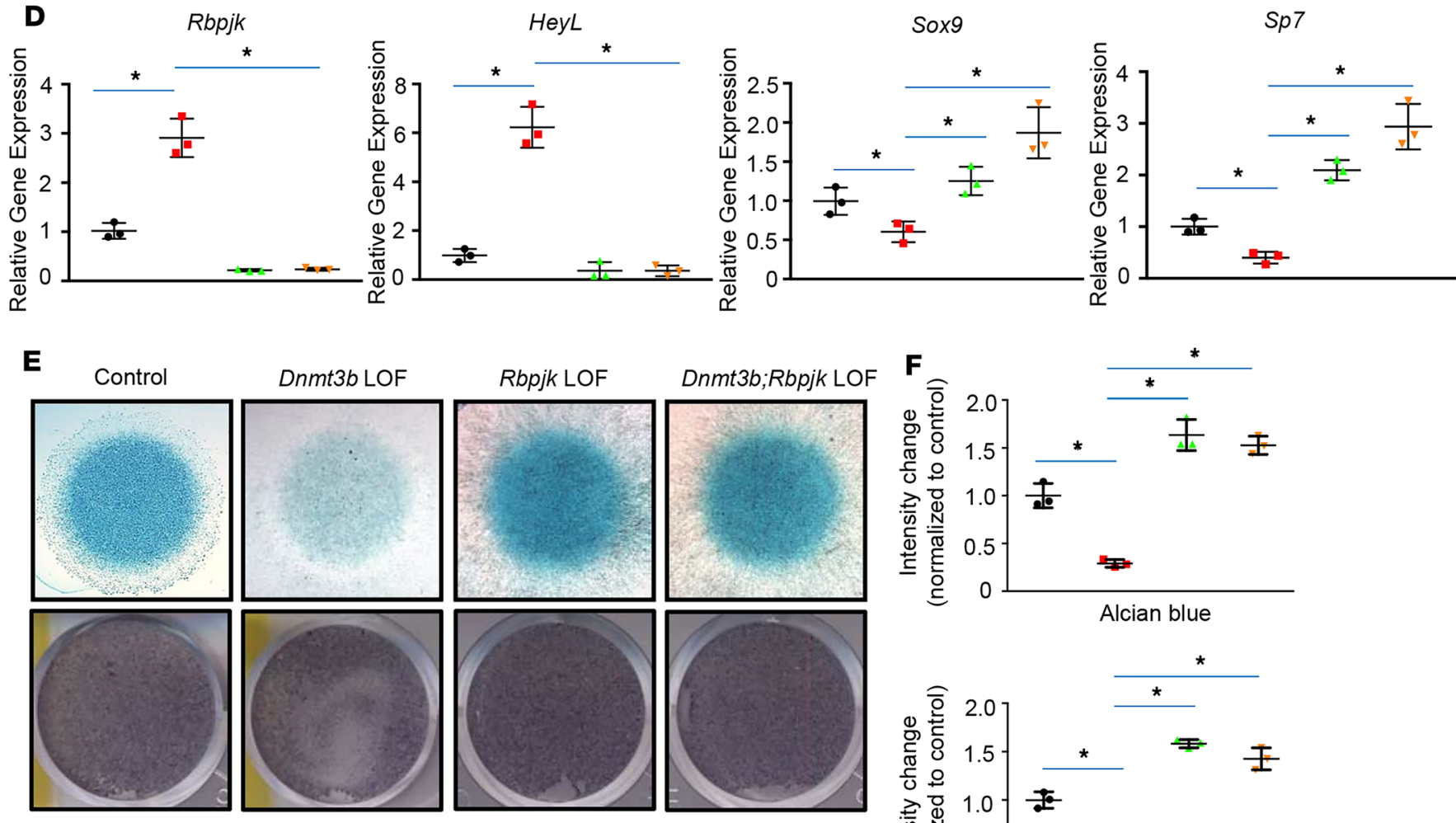

$$
\begin{array}{ll}
\text { - Control } & \Delta \text { Rbpjk LOF } \\
\text { Dnmt3b LOF } & \nabla \text { Dnmt3b; Rbpjk LOF }
\end{array}
$$
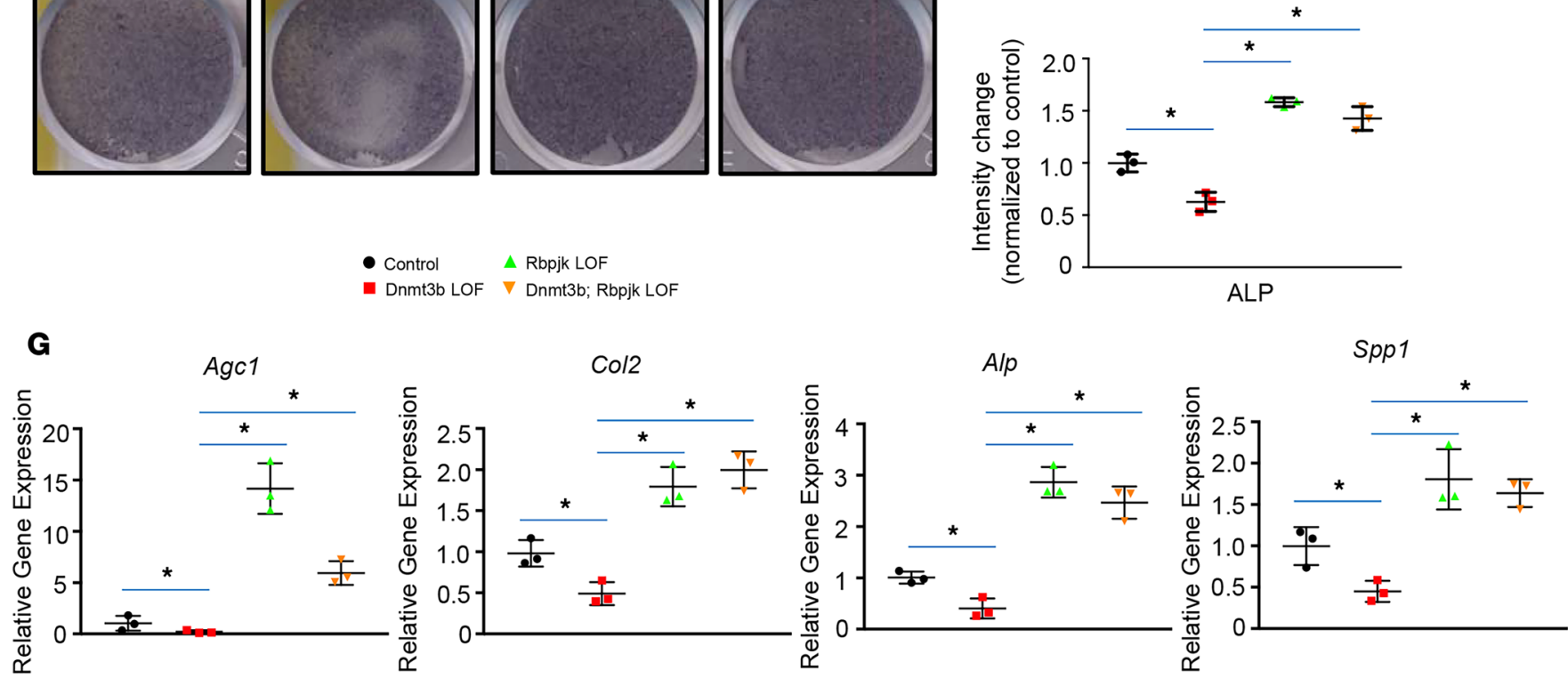

Figure 5. Rbpjк is a target of Dnmt3b. (A) A schematic representing 2 Dnmt3b binding sites (CpC islands, green box) identified in Rbpjк gene. (B) Pulldown of genomic DNA with a Dnmt3b antibody (ChIP assay) shows interaction of Dnmt3b with its binding site in the Rbpjк promoter region by qPCR utilizing specific primers $(n=3)$. (C) Rbpjk promoter luciferase assay was performed in C3H10T1/2 cells with Dnmt3b loss of function (LOF) and gain of function 
(GOF) ( $n=3)$. (D) Real-time qPCR analysis of Rbpjk, HeyL, Sox9, and Sp7 in C3H10T1/2 cells with Dnm3b LOF and Rbpjк LOF. The mRNA levels were normalized to that of $A c t b$ and then were normalized to the control group $(n=3)$. (E) Alcian blue and alkaline phosphatase (ALP) stainings were performed to examine the chondrogenic and osteogenic processes, respectively $(n=3)$. (F) Alcian blue and ALP intensity was measured using NIH ImageJ software ( $n$ = 3). (G) Real-time qPCR analyses were performed to determine the relative expression of Agc1, Col2, Alp, and Spp1 in C3H10T1/2 cells. The mRNA levels were normalized to that of Actb and then were normalized to the control group. Data are presented as mean \pm SD. ${ }^{*} P<0.05$ by 1 -way ANOVA test.

via DAPT treatment could enhance fracture repair through promoting MPC differentiation in wildtype mice $(3,28)$. To further validate if Rbpjк-mediated Notch pathway is the critical downstream target of Dnmt3b in the context of fracture healing in mice, we first employed DAPT, the Notch inhibitor, to suppress Notch activation in cell cultures and in $D n m t 3 b^{P r x l}$ mice. Similar to Rbpjк inhibition, Notch inhibition via DAPT in limb bud-derived MPCs substantially increased chondrogenic differentiation and cartilage matrix deposition, as reflected by enhanced $\mathrm{ABH}$ staining in $D n m t 3 b^{P r x I}$ micromass cell cultures following DAPT treatment (Supplemental Figure 6, A and B). In accordance with these observations, the expression of chondrogenic regulatory gene, Sox9, and the cartilage-specific extracellular matrix molecules, Col2al and $A g c 1$, were induced with expression levels higher than those observed in littermate controls under basal conditions (Supplemental Figure 6C). In bone marrow-derived MPC cultures, Notch inhibition with DAPT had a similar effect. DAPT treatment significantly stimulated the osteogenic differentiation and osteoblast mineral deposition in bone marrow-derived MPCs of Dnmt $3 b^{P r x 1}$ mice (Supplemental Figure 6, D and E). Real-time qPCR revealed increased expression of osteogenic genes, including Runx2 and Spp1, in bone marrow-derived MPCs of DAPT-treated $D_{n m t} 3 b^{\text {Prxl }}$ mice (Supplemental Figure $6 \mathrm{~F}$ ). We then treated control mice and $D n m t 3 b^{\text {Prxl }}$ mice with a single dose of DAPT (50 mg/kg body weight) $2 \mathrm{dpf}$. Consistent with our previous finding (28), DAPT treatment significantly downregulated Notch pathway in both control mice and $D n m t 3 b^{\text {Prx }}$ mice, as reflected by decreased Notch downstream genes, such as HeyL and Hey1 (Figure 6A). As expected, DAPT treatment stimulated cartilage tissue formation $10 \mathrm{dpf}$ and bone tissue formation $14 \mathrm{dpf}$ in control mice. More importantly, DAPT treatment also reversed chondrogenic and osteogenic differentiation defect in $D n m t 3 b^{P r x I}$ mice during fracture healing. Histological analyses revealed an accelerated endochondral ossification process, including increased cartilage tissue $10 \mathrm{dpf}$ and bone tissue $14 \mathrm{dpf}$ in DAPT-treated $D n m t 3 b^{P r x I}$ mice (Figure 6, B and C). Furthermore, micro-CT analyses of mineralized calluses confirmed that DAPT treatment induced a robust bone formation in fractures of $D n m t 3 b$ ${ }^{P r x}$ mice, shown as increased bony callus volume 10 and $14 \mathrm{dpf}$, compared with the DMSO-treated group. DAPT treatment restored the bony callus formation in $D n m t 3 b^{\text {Prx }}$ mice to a level comparable to that in DAPT-treated control mice (Figure 6, D and E). Interestingly, we also observed increased osteoclast activity in fractures of DAPT-treated $D n m t 3 b^{P r x 1}$ mice $14 \mathrm{dpf}$ compared with fractures of DMSO-treated Dnmt3b ${ }^{\text {Prx }}$ mice (Supplemental Figure 7, A and B). Altogether, the results show that Dnmt3b induces chondrogenic and osteogenic differentiation in MPCs through a mechanism involving reduced Notch signaling and its expression is required for normal bone regeneration.

\section{Discussion}

Bone fracture repair occurs through a complex and integrated series of events involving several transcriptional responses unique to different cell types, including MPCs, chondrocytes, osteoblasts, and osteoclasts. The recruitment and differentiation of endogenous progenitor cells to the fracture site is a key initial step in fracture healing. Although the cellular and molecular events regulating differentiation during bone regeneration have been studied in various cell populations and animal models (29), the epigenetic factors regulating regeneration are largely unknown. Recent epigenome screening of human fracture tissues suggests that DNA methylation is involved in bone healing (14). Consistently, we showed a reduced Dnmt3b expression in fracture calluses of HFD-induced diabetic mice and aging mice. Thus, the present work is timely and reveals the critical effect of Dnmt3b LOF on regulating MPC differentiation and bone repair.

We have previously established that Dnmt3b is the most regulated DNA methyltransferases during the endochondral bone regeneration process (20). Dnmt3b expression is induced in the early repair phase and is highly expressed in progenitor cell populations (20). In the present study, we provided additional evidence and demonstrated that Dnmt3b positively regulates chondrogenic and osteogenic differentiation of MPCs by using well-established primary embryonic limb bud-derived mesenchymal cells and bone marrow-derived mesenchymal cells $(30,31)$. In both cells, key transcription factors involved in chondrogenic and osteogenic 


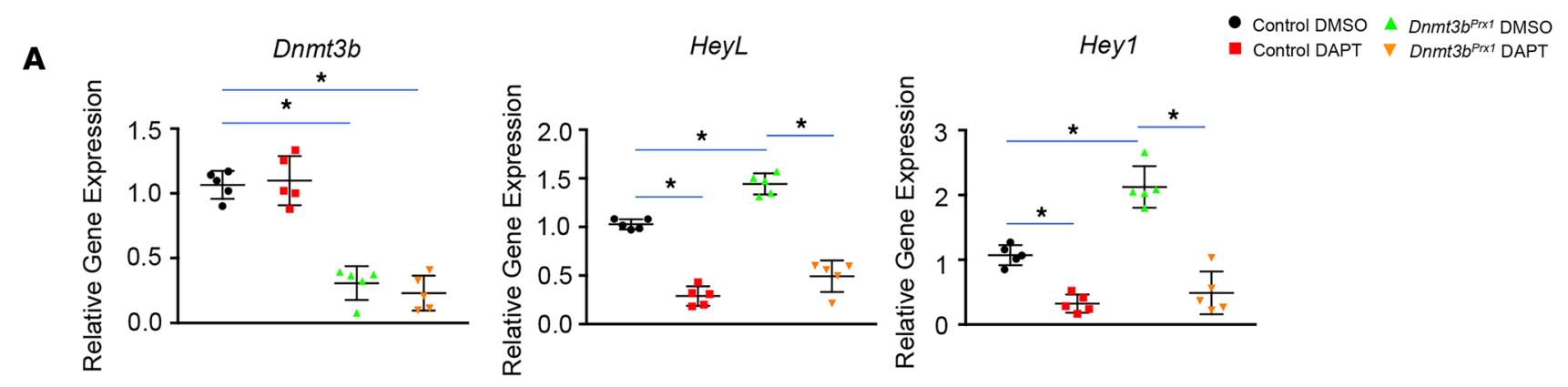

B

Control

Dnmt3bPrx1
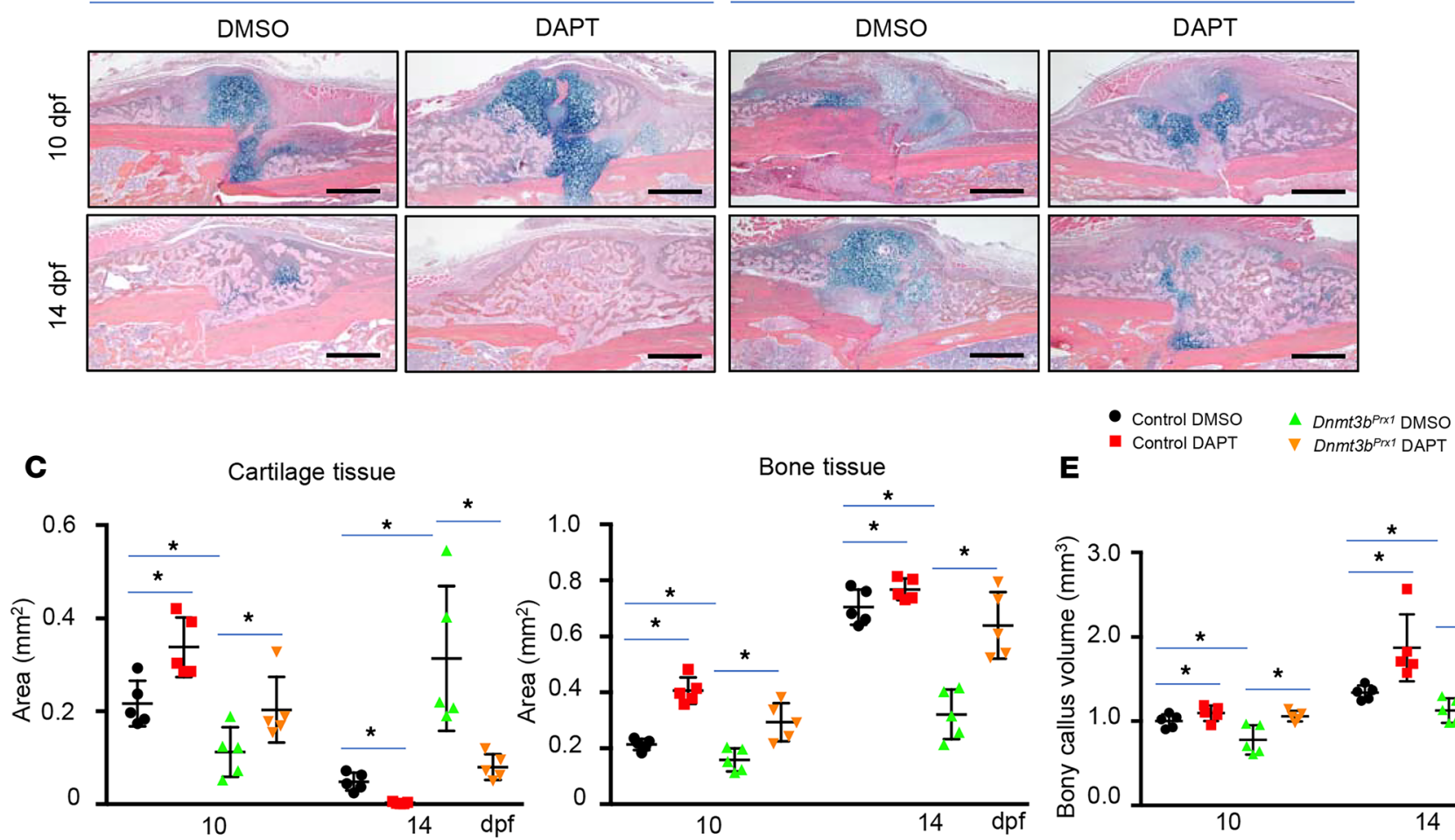

D

Control

$D n m t 3 b^{\text {Prx } 1}$
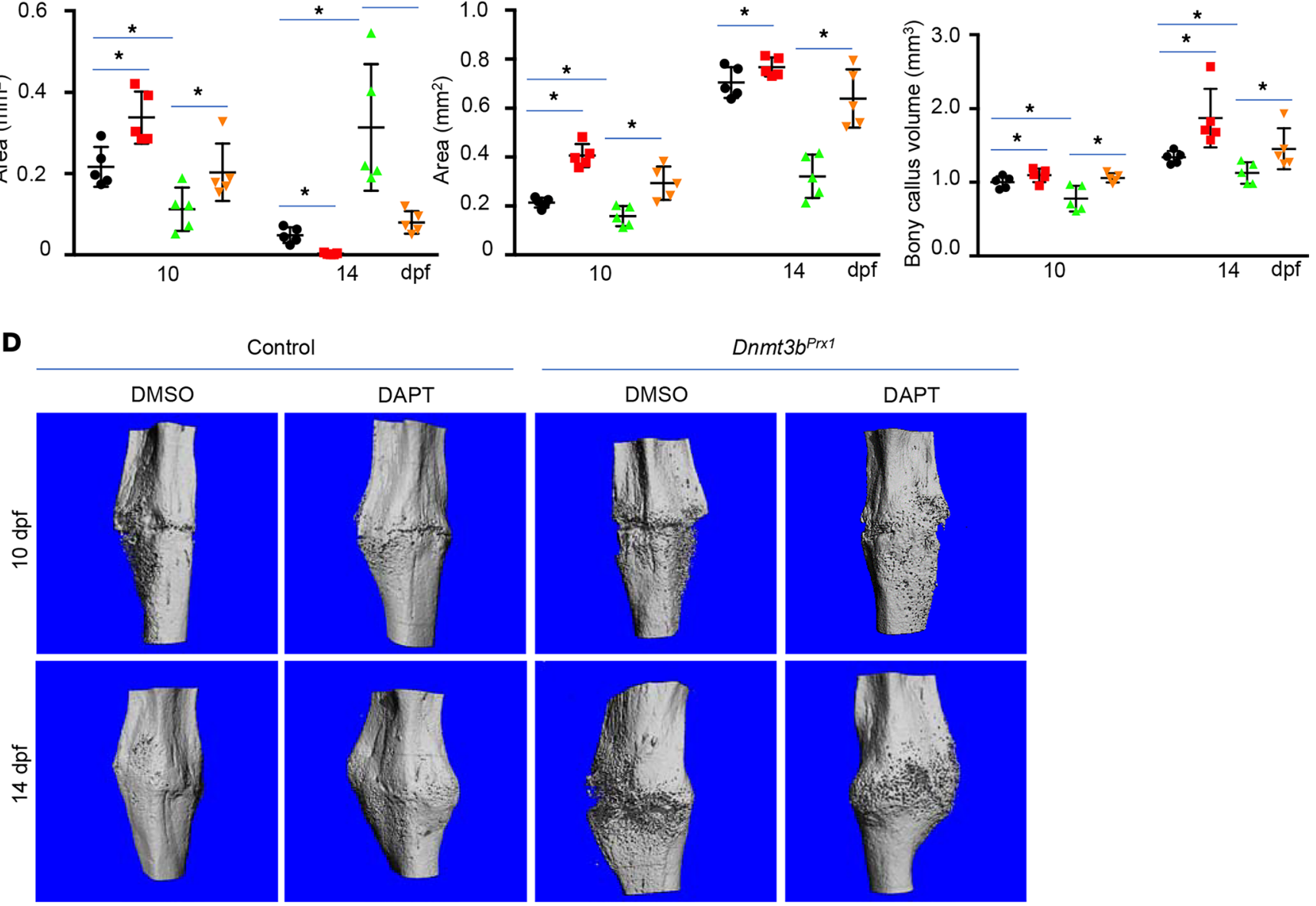
Figure 6. Notch inhibition via dual antiplatelet therapy restored fracture repair in Dnmt $\mathbf{b b}^{\text {prx }}$ mice. (A) Notch pathway inhibition efficacy was determined by real-time qPCR. The mRNA expression of Dnmt3b, HeyL, and Hey1 was measured in fracture calluses of dual antiplatelet therapy-treated (DAPT-treated) and DMSO-treated control mice and $D n m t 3 b^{P_{r x x}}$ mice 3 days after fracture (dpf). (B) Alcian blue/Hematoxylin/Orange $G$ (ABH/OG) staining of fracture callus sections of DAPT- and DMSO-treated control and Dnmt3 $b^{P_{r x} 1}$ mice at the indicated times $(n=5)$. Scale bar: $500 \mu \mathrm{m}$. (C) Histomorphometry quantification of the cartilage and bone areas was performed on ABH/OG-stained fracture callus sections $(n=5)$. (D) Microcomputed tomography assessment of mineralized bone in fracture calluses of DAPT- and DMSO-treated control mice and Dnmt36 $b^{P_{r \times 1}}$ mice at the indicated times $(n=5)$. (E) Quantification of bony callus volumes 10 and $14 \mathrm{dpf}(n=5)$. Data are presented as mean \pm SD. ${ }^{*} P<0.05$ by 2 -way ANOVA test.

differentiation, $S o x 9$ and $S p 7$, respectively, were reduced by loss of Dnmt3b. In contrast, cell proliferation and apoptosis (less than 1\%; data not shown) are not affected in MPC cultures, indicating that decreased progenitor cell differentiation capacity but not cell viability or impaired proliferation accounts for the reduced fracture callus formation. The findings were consistent with in vivo fracture experiments that demonstrated decreased chondrogenic and osteogenic differentiation and impaired fracture healing in Dnmt $3 b^{\text {Prx }}$ mice. In addition to reduced secondary osteogenesis in association with the cartilaginous callus, there was also decreased osteogenesis along the periosteal bone surface adjacent to the fracture site where primary bone formation occurs, suggesting a direct cell autonomous effect on osteoblast differentiation (22). We previously reported delayed bone remodeling in fractures of mice with conditional $\mathrm{Agcl}$-Cre-mediated chondrocyte gene deletion (20). In the current study, we similarly show delayed bone remodeling in fractures with somatic Dnmt3b LOF in limb budderived MPCs, with reduced numbers of osteoclasts in the early remodeling phase (14 dpf), but persistence of osteoclasts at later times (21 and $28 \mathrm{dpf}$ ) when remodeling had been completed in fractures of control mice. As the Prx1-driven gene deletion does not directly affect osteoclast precursors due to lack of Prx1 expression in these cells, the findings suggest that the reduced osteoclast differentiation is secondary to noncell autonomous signals from chondrocytes and/or osteoblasts. Finally, biomechanical testing, the most sensitive method to determine fracture healing, confirmed the impaired fracture healing in mice with Dnmt3b LOF in MPCs.

DNA methylation is highly responsive to environmental stimuli as it broadly regulates the expression of growth factors, receptors, and transcription factors and coordinates signaling networks at the genome level $(32,33)$. TGF- $\beta$, BMP, Wnt/ $\beta$-catenin, Notch, and IHH signaling pathways are established downstream targets of DNA methylation (34-39). In current study, we demonstrated for the first time to our knowledge that the Rbpjк-mediated Notch pathway is regulated via epigenetic alteration in the context of fracture repair. In vitro cell culture experiments identified Rbpjк as a direct downstream target of Dnmt $3 b$ since hypomethylation of the Rbpjк promoter and gene body occurred with loss of Dnmt $3 \mathrm{~b}$. This resulted in the upregulation of Rbpjк gene expression and an increase in Notch signaling in MPCs. Moreover, the 2 Dnmt3b-binding regions in Rbpjк (CpG islands) were found to be correlated with active histone modification in the University of California, Santa Cruz genome browser, confirming that the 2 CpG islands are the important epigenetic regulatory loci (40). Importantly, Rbpjк inhibition via lenti-shRNA restored chondrogenesis and osteogenesis in MPCs with Dnmt3b LOF, indicating the functional importance of

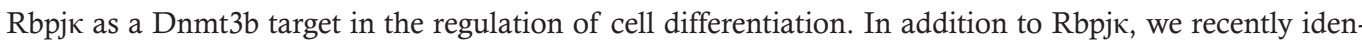
tified hypomethylation in Cxcl12 gene in Dnmt3b LOF chondrocytes (20), suggesting Dnmt3b LOF could directly modify DNA methylation pattern in chondrocyte maturation-related genes. Findings from other groups also demonstrated that chondrogenic- and osteogenic-related genes could be regulated via alteration of DNA methylation in promoters (41-43). We thus speculate Dnmt3b LOF leads to progenitor differentiation defect through a combination of an altered Notch pathway and the alteration of DNA methylation in chondrogenic- and osteogenic-specific genes. A whole-genome bisulfite DNA methylation sequencing will be extremely helpful to probe all the differential methylated loci and comprehensively understand the role of Dnmt3b-mediated epigenetic alteration in the regulation of progenitor differentiation.

To confirm Rbpjк as a Dnmt3b target in the context of fracture repair in vivo, we then treated Dnmt $3 b^{\text {Prx }}$ mice with a single dose of DAPT 2 dpf. Consistent with in vitro findings, transient inhibition of Notch by DAPT at least partially restored MPC differentiation and fracture repair in $D n m t 3 b^{P r x l}$ mice. Although we have previously demonstrated that a single dose of DAPT was sufficient to suppress Rbpjк-mediated Notch pathway in wildtype mice, with minimal side effects on progenitor cell recruitment during hematoma phase (28), it is still worth optimizing the injection regimen of DAPT to minimize its potential toxicity (44-46) and incessantly promoting the differentiation of progenitor cells, which can be continuously recruited to injury site via reestablished blood vessels and from adjacent periosteum tissue during the cartilaginous and bony callus phase (47). Furthermore, to fully elucidate the interaction between Dnmt $3 \mathrm{~b}$ and Rbpjк in the context of progenitor cell differentiation and 
fracture repair, it would be better to generate Rbpjк LOF mice under Dnmt3b LOF background since DAPT is not an Rbpjк-specific inhibitor. DAPT can block $\gamma$-secretase and thus suppresses both canonical and noncanonical Notch pathway (48). Given that sustained inhibition of Rbpjк using Prx1Cre led to severe osteoporosis in adult mice and led to fracture nonunion primarily because ablation of Rbpjк exhausted the progenitor population during skeletal development $(3,23,49)$, doxycycline-induced ten-eleven translocation (Tet) system (50)

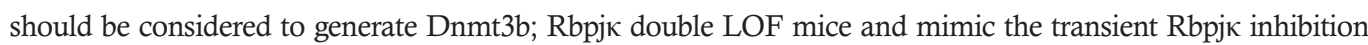
effect. Primary MPCs isolated from these animals will provide more robust cellular and molecular insights into the mechanisms involved in the epigenetic regulation of MPC differentiation.

In the current study, we performed fracture surgeries on 10-week-old mice and confirmed the positive role of Dnmt3b in stimulating fracture repair in mice. Although 10-week-old mice are skeletally mature, they are relatively young animals for fracture studies, with robust healing capacity. Aged mice will experience more advanced fracture healing delay $(51,52)$ and are more clinically relevant. Clinical studies of patients have documented that aging and chronic diseases, in particular inflammatory conditions, compromise the normal healing process and sometimes lead to fracture nonunion by negatively affecting MPC differentiation (29, 53-58). Therefore, it is worth examining the effect of Dnmt3b gain of function and Rbpjк LOF in mice under aging or systemic inflammatory conditions, to provide novel cellular and molecular candidates for therapeutic intervention. Additionally, Dnmt3a is the other critical de novo enzymes, which has been reported to work synergistically with Dnmt3b to regulate events during embryonic development, for example, HSC differentiation (18). Double knockout of Dnmt3a and Dnmt3b resulted in enhanced HSC self-renewal and blockage of cell differentiation, whereas single deletions of $D n m t 3 a$ and $D n m t 3 b$ results in a mild phenotype $(17,18)$, indicating the overlap role of Dnmt3a and $3 b$ in regulating HSC differentiation. Similarly, Dnmt $3 b^{P x x}$ mice display a mild limb phenotype in embryos (data not shown), and no gross bone phenotype is observed in adult animals, likely due to the compensatory effect of Dnmt3a or other DNA methylation enzymes in MPCs under physiology conditions. Therefore, it is worth determining the role of Dnmt3a and other demethylation enzymes, such as Tet, enzymes in the regulation of skeletal tissue homeostasis under challenging conditions.

In summary, we have defined a key role for Dnmt $3 b$ as a regulator of normal fracture repair. In normal fracture healing, Dnmt3b expression is increased, and this is associated with reduced Notch signaling and robust MPC differentiation along the chondrogenic and osteogenic bone-forming lineages. At the molecular level, we found that Dnmt3b deletion induced Rbpjк expression, and the subsequent Notch activation inhibited MPC differentiation. Future studies will identify conditions in which altered Dnmt3b expression and DNA methylation lead to compromised fracture repair. We anticipate that Notch inhibition represents a potential new therapy for fracture repair defects associated with Dnmt3b LOF-induced Notch activation in MPCs.

\section{Methods}

Mice. Dnmt $3 b^{\text {Prx }}$ mice were generated by breeding $D n m t 3 b^{f f}$ mice (59) (obtained from the Mutant Mouse Regional Resource Center, Davis, California, USA; 29887) with Prx1-Cre transgenic mice (60) (purchased from Jackson Laboratory; 005584). Dnmt $3 b^{P r x I}$ mice were viable, fertile, and produced at expected Mendelian ratios. Unilateral tibial fracture procedures (20) were performed on 10-week-old Dnmt $3 b^{\text {Prx }}$ mice and their littermate controls. One dose of DAPT $(50 \mathrm{mg} / \mathrm{kg}$; Enzo Life Science, catalog ALX-270416) or DMSO (vehicle) was given to $D n m t 3 b^{P r x I}$ mice via intraperitoneal injection 2 days after fracture to suppress Notch pathway.

Histological analyses of fracture callus. The fractured tibia samples were collected for histological analyses 7 , $10,14,21$, and $28 \mathrm{dpf}$ ( $n=5$ per group in each time point). After mice were euthanized, the fractured tibiae were collected along with the adjacent tissues and fixed in $10 \%$ neutral buffered formalin (NBF) for 3 days and then decalcified for 10 days in $14 \%$ ethylenediaminetetraacetic acid solution ( $\mathrm{pH} 7.2)$. Tissue was then sectioned longitudinally at a thickness of $5 \mu \mathrm{m}$. ABH/OG staining and TRAP staining were performed to analyze the callus composition and osteoclast formation in the fracture region. Sections were visualized with nanozoomer (NanoZoomer HT). Histomorphometric analyses were performed using NIH ImageJ software 1.46r (Wayne Rasband). Mesenchyme area, cartilage area, bone area, and osteoclast surface per bone surface (Oc. S/BS) were measured and calculated as previously described (28). In general, mesenchyme tissue had a fibroblastic appearance at the margin of fracture and was stained in light blue. Cartilage tissue comprised rounded-shaped chondrocytes and was stained in dark blue. Bony tissue was stained in red. IHC staining for Dnmt3b (Abcam, ab16049, 1:1000) was performed on paraffin sections following the antigen retrieval and colorimetric development methodologies. Citrate buffer with high temperature and pressure antigen retrieval was used. 
Micro-CT analysis. After careful dissection and removal of the intramedullary pins in fractured tibiae, we examined the fracture calluses using the micro-CT (VivaCT 40, Scanco) scanner at $10.5 \mu \mathrm{m}$ resolution (55 kV, $145 \mu \mathrm{A}, 300 \mathrm{~ms}$ integration time). Six hundred slices $(6.3 \mathrm{~mm})$ centered on the midpoint were used for micro-CT analyses. A contour was drawn around the margin of the entire callus and a lower threshold of 150 per mille was then applied to segment mineralized tissue (all bone inside the callus). A higher threshold of 460 per mille was applied to segment the original cortical bone inside the callus volume. Quantification for the volumes of the bone calluses was determined as previously described using the Scanco analysis software ( $n=6$ per group in each time point) (61). 3D images were generated using a constant threshold of 240 per mille for the diaphyseal callus region of the fractured tibia. The intact tibia samples from 10 -week-old control mice and $D n m t 3 b^{P x x}$ mice were also scanned at resolution $21 \mu \mathrm{m}(70 \mathrm{kV}, 114 \mu \mathrm{A}, 300 \mathrm{~ms}$ integration time $)$. Cortical bone was assessed at a region of interest centered $5 \mathrm{~mm}$ proximal to the distal tibiofibular junction, spanning 50 slices $(1 \mathrm{~mm})$. Trabecular bone was assessed at the proximal metaphysis, distal of the growth plate, and spanned 200 slices (4.2 $\mathrm{mm}$ ).

Biomechanical torsion testing. The fractured tibia bone samples harvested $28 \mathrm{dpf}$ ( $n=10$ per group) were potted with methacrylate (MMA) bone cement (Lang Dental Manufacturing) and secured in 1.2-cm-long cylinders (6-mm diameter) to keep the fracture sites in the midinterval. After the MMA solidified, the bone samples with the cylinders were set up on the torsion machine and processed using the custom LabVIEW (National Instruments) program. The data of torsion testing were present against the rotational deformation as the maximum torque, torsional rigidity, and energy to fracture using the custom MATLAB 2017b program (Mathworks).

Limb bud-derived mesenchymal cell isolation and micromass culture. Limb bud-derived MPCs were isolated from E11.5 Dnmt3b ${ }^{P x x}$ embryos and control embryos (25). Briefly, the limb buds from embryos were dissected and digested with Trypsin (Life Technologies; 25200056) at $37^{\circ} \mathrm{C}$ for 5 minutes. MPC cell suspensions were filtered through a $0.4-\mu \mathrm{m}$ cell strainer and reconstituted to the density at $2 \times 10^{7} \mathrm{cells} / \mathrm{mL}$. MPC cell suspensions $(10 \mu \mathrm{l})$ were seeded in the center of 24 -well plates for 1.5 hours and then cultured in high-glucose DMEM supplemented with 10\% FBS (Life Technologies, catalog 26140079). For chondrogenic differentiation, the micromasses were cultured with maturation medium, including $10 \mathrm{mM} \beta$-glycerophosphate (Sigma-Aldrich, catalog G9422) and $50 \mu \mathrm{g} / \mathrm{mL}$ ascorbic acid (Sigma-Aldrich, catalog A4544) for 3 days. We also used $10 \mu \mathrm{M}$ DAPT(Calbiochem, catalog 565784 ) as a $\gamma$-secretase inhibitor to suppress Notch pathway during the micromass culture. Alcian blue staining was performed on micromasses to assess the chondrogenic capacity of MPCs in Dnmt $3 b^{P r x 1}$ embryos and control embryos. After 30 minutes fixation with $10 \%$ NBF, the micromass cultures were stained with $1 \%$ Alcian blue solution for 30 minutes and then rinsed with 70\% ethanol. After washing 3 times with $\mathrm{dd}_{2} \mathrm{O}$, images were captured by scanner (EPSON, Perfection 1660).

Bone marrow-derived mesenchymal cells isolation and culture. Bone marrow-derived MPCs were isolated from 10-week-old Dnmt3 $b^{P r x I}$ mice and control mice. Both femur and tibia were dissected, and bone marrow-derived cells were centrifuged into the $1.5-\mathrm{mL}$ tube from the marrow cavity. MPCs were then seeded on a 100-mm tissue culture dish in MEM- $\alpha$ (Life Technologies, catalog A1049001) containing 10\% FBS (Gibco, catalog 16000044), and 1\% Penicillin-Streptomycin (Thermo Fisher, catalog 15140122). Nonadherent cells were removed after 3 days of culture and MPCs were cultured and expanded until 70\% confluency. The cells $\left(4 \times 10^{5}\right.$ cells per well) of $D n m t 3 b^{P r x l}$ mice and control mice were plated for 14 days without change of mouse MSC medium (StemCell Technologies, catalog 05513) for colony-forming unit assay. Crystal violet staining was performed and type I colonies were quantified as previously described (23). For osteogenic differentiation, MPCs were seeded at a density of $4 \times 10^{5}$ cells $/ \mathrm{mL}$ in osteogenic medium, including $10 \mathrm{mM} \beta$-glycerophosphate and $50 \mu \mathrm{g} / \mathrm{mL}$ ascorbic acid. We also used $10 \mu \mathrm{M}$ DAPT (Calbiochem; 565784) as a $\gamma$-secretase inhibitor to suppress Notch pathway during osteogenic differentiation. ALP staining and Alizarin red staining were performed on cell cultures to assess the osteogenic capacity of MPCs from $D n m t 3 b^{P r x l}$ and control mice at indicated time points. Briefly, after $10 \%$ NBF fixation, the cell cultures were stained with the NBT/BCIP substrate solution (ccccg) to detect ALP activity or alizarin red solution (Sigma-Aldrich, catalog A5533) to detect mineral deposition. After washing 3 times with $\mathrm{H}_{2} \mathrm{O}$, images were captured by scanner (EPSON, Perfection 1660).

C3H10T1/2 cell culture and differentiation. C3H10T1/2 cells (ATCC CCL-226) were infected with Lenti-shDnmt3b virus (multiplicity of infection [MOI] $=50$, Origene, catalog TL508059V) and Lenti-shRb-

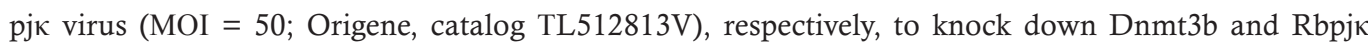
expression in cells. $\mathrm{C} 3 \mathrm{H} 10 \mathrm{~T} 1 / \mathrm{c}$ cells were cultured in chondrogenic medium for 14 days and osteogenic medium for 7 days. Alcian blue and ALP stainings were used to examine the chondrogenic and osteogenic differentiation processes. 
Table 1. Primer sequences for real-time qPCR

\begin{tabular}{|c|c|}
\hline Genes & Sequences \\
\hline \multirow{2}{*}{ Dnmt3b } & 5'-AATACCCAACTCCTTCACCAC-3' \\
\hline & 5'-TCTTCACTACTCATCCTCACCT-3' \\
\hline \multirow{2}{*}{ Sox9 } & 5'-CGGCTCCAGCAAGAACAAG-3' \\
\hline & 5'-TGCGCCCACACCATCA-3' \\
\hline \multirow{2}{*}{ Col2a1 } & 5'-GCAGAGATGGAGAACCTGGTA-3' \\
\hline & 5'-AGCCTTCTCGTCATACCCT-3' \\
\hline \multirow{2}{*}{ Agc1 } & 5'-CGTGTTTCCAAGGAAAAGGA-3' \\
\hline & 5'-TGTGCTGATCAAAGTCCA G-3' \\
\hline \multirow{2}{*}{ Sp7 } & 5'-CTTCTTTGTGССTССTTTCС-3' \\
\hline & 5'-GCGTCСTСTCTGСTTCA-3' \\
\hline \multirow{2}{*}{ Runx2 } & 5'-CGTCCACTGTCACTTTAATAGCTC-3' \\
\hline & 5'-GTAGCCAGGTTCAACGATCTG-3' \\
\hline \multirow{2}{*}{$A l p$} & 5'-TСАССТTСТСТССТССАТСС-3' \\
\hline & 5'-CTTCCTGGGAGTCTCATCCT-3' \\
\hline \multirow{2}{*}{ Spp1 } & 5'-TCGTCATCATCGTCGTCCA-3' \\
\hline & 5'-AGAATCCTGTGTCCTCTCAAG-3' \\
\hline \multirow{2}{*}{ HeyL } & 5'-CAGCCCTTCGCAGATGCAA-3' \\
\hline & 5'-CCAATCGTCGCAATTCAGAAAG-3' \\
\hline \multirow{2}{*}{ Hey1 } & 5'-GCGCGGACGAGAATGGAAA-3' \\
\hline & 5'-TCAGGTCATCCACAGTCATCTG-3' \\
\hline \multirow{2}{*}{ Actb } & 5'-AGATCTGGATCAGCAAGCAG-3' \\
\hline & 5'-GCGCAAGTTAGGTTTTGTCA-3' \\
\hline
\end{tabular}

ChIP assay. C3H10T1/2 cells $\left(1 \times 10^{7}\right)$ were harvested and fixed with formaldehyde for ChIP assays. Chromatin was fragmented by sonication to shear DNA to 200-500 bp in size. Chromatin fragments were immunoprecipitated using the Dnmt3b antibody (1:50, Abcam, ab2851), whereas Histone $\mathrm{H} 3$ and IgG antibodies were used as positive and negative controls, respectively. After reverse crosslinking of protein-DNA, the enrichment of $R b p j \kappa$ DNA fragment during immunoprecipitation was analyzed by quantitative real-time PCR. Primer sequences used for CpG Island 1 in Rbpjк promoter were forward, 5'-CTAGGTGACTCAGATGCATGAC-3', and reverse, 5'-TCCCGAGCCTGGTACTT-3'; primer sequences for CpG Island 2 in Rbpjк gene body were forward, 5'-CCTTTGTTTCGCCGCCTTA-3', and reverse, 5'-CCAGCTACTGAAGAGAGGGATA-3'.

Methylation $q P C R$. Methylation qPCR for Rbpjк was performed according to the manufacturer's instruction (QIAGEN). Genomic DNA isolated from limb bud-derived MPCs in Dnmt3b LOF and control mice was enzymatically digested by methylation-sensitive or methylation-dependent enzyme separately. The enzyme-digested genomic DNA was then used for qPCR and the primers for Rbpjк methylation (CpG Island 106810 and 106811) were obtained from QIAGEN (EPMM106810 and EPMM106811).

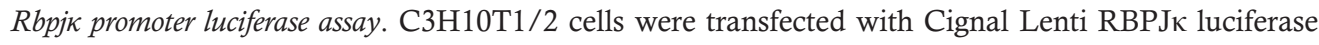
reporter (QIAGEN) with MOI of 5 and then cultured under $2 \mu \mathrm{g} / \mathrm{mL}$ puromycin selection. The stable C3H10T1/2 cells were transfected with lentivirus to inhibit or overexpress Dnmt3b for 24 hours before performing the luciferase assay.

Real-time qPCR and Western blot. RNA was isolated from primary cell cultures or C3H10T1/2 cells using RNeasy Mini Kit (QIAGEN). RNA was also isolated from fracture calluses of HFD-induced mice and 18-month-old wild-type mice $7 \mathrm{dpf}$ as well as fracture calluses of DMSO- and DAPT-treated control mice and $D n m t 3 b^{P r x I}$ mice $3 \mathrm{dpf}$. Four millimeters of fracture calluses were flushed of morrow and homogenized for mRNA extraction. cDNA synthesis and real-time RT-PCR was performed according to the manufacturers' instructions. Primer sequences for Dnmt3b, Sox9, Col2a1, Agc1, Sp7, Runx2, Spp1, Alp, HeyL, Hey1, and $A c t b$ are detailed in Table 1 . Western blot analyses were performed on the protein lysates from limb bud-derived or bone marrow-derived MPCs. The following primary antibodies were used: Rbpjк (Cell Signaling, 5313, 1:1000) and Actb (Sigma-Aldrich, 2228, 1:2000). Quantification of the protein expressions (relative intensity) was measured using NIH ImageJ 1.46r software. 
Statistics. Statistical analyses were performed using GraphPad Prism. Comparisons between 2 groups were performed using 2-tailed, unpaired Student's $t$ test and 1- or 2-way ANOVA followed by Tukey test to compare multiple groups under different genotypes or under different treatments at multiple time points. All data are presented as the mean \pm SD. $P$ values of less than 0.05 were considered statistically significant.

Study approval. Unilateral tibial fracture procedures were performed in compliance with Washington University's Institutional Animal Care and Use Committee, accredited by the Association for Assessment and Accreditation of Laboratory Animal Care International, and the NIH guidelines.

\section{Author contributions}

JY, TX, CW, YA, RJO, and JS designed the study. JY, TX, and CW conducted the study. JY, TX, and CW collected the data. JY, TX, and CW analyzed the data. JY, TX, CW, HJ, PT, JG, YA, RJO, and JS interpreted the data. JY, TX, CW, YA, RJO, and JS drafted the manuscript. As co-first authors, JY and TX contributed equally to the project. JY was more involved in manuscript preparation; thus, his name is in front of TX's.

\section{Acknowledgments}

This work was supported by the following NIH grants: R01 grants (AR075860 to JS, AR069605 to RJO and AR049192, AR054326, AR072623 to YA); Shriners grant 86200 to YA; a T32 training grant that supported CW (AR060719 to Roberto Civitelli); and a P30 Core Center Grant (AR074992 to Musculoskeletal Research Center). The fracture calluses from HFD-induced mice and 18-month-old aging mice were a gift from Lianping Xing's lab (School of Medicine, University of Rochester, Rochester, New York, USA). Bo Zhang (Department of Developmental Biology, Washington University in St. Louis) helped with statistical analysis.

Address correspondence to: Jie Shen, Department of Orthopaedic Surgery, Washington University in St. Louis, 660 South Euclid, CB 8233, St. Louis, Missouri 63110, USA. Phone: 314.747.2567; Email: shen.j@wustl.edu.

1. Einhorn TA, Gerstenfeld LC. Fracture healing: mechanisms and interventions. Nat Rev Rheumatol. 2015;11(1):45-54.

2. Court-Brown CM, Caesar B. Epidemiology of adult fractures: A review. Injury. 2006;37(8):691-697.

3. Wang C, et al. NOTCH signaling in skeletal progenitors is critical for fracture repair. J Clin Invest. 2016;126(4):1471-1481.

4. Schmidt-Bleek K, Kwee BJ, Mooney DJ, Duda GN. Boon and bane of inflammation in bone tissue regeneration and its link with angiogenesis. Tissue Eng Part B Rev. 2015;21(4):354-364.

5. Yuasa M, et al. Fibrinolysis is essential for fracture repair and prevention of heterotopic ossification. J Clin Invest. 2015;125(8):3117-3131.

6. Murao H, Yamamoto K, Matsuda S, Akiyama H. Periosteal cells are a major source of soft callus in bone fracture. J Bone Miner Metab. 2013;31(4):390-398.

7. Gerstenfeld LC, Cullinane DM, Barnes GL, Graves DT, Einhorn TA. Fracture healing as a post-natal developmental process: molecular, spatial, and temporal aspects of its regulation. J Cell Biochem. 2003;88(5):873-884.

8. Maes C, et al. Placental growth factor mediates mesenchymal cell development, cartilage turnover, and bone remodeling during fracture repair. J Clin Invest. 2006;116(5):1230-1242.

9. Kronenberg HM. Developmental regulation of the growth plate. Nature. 2003;423(6937):332-336.

10. Einhorn TA. The cell and molecular biology of fracture healing. Clin Orthop Relat Res. 1998;(355 suppl):S7-S21.

11. Ferguson C, Alpern E, Miclau T, Helms JA. Does adult fracture repair recapitulate embryonic skeletal formation? Mech Dev. 1999;87(1-2):57-66

12. Vortkamp A, Pathi S, Peretti GM, Caruso EM, Zaleske DJ, Tabin CJ. Recapitulation of signals regulating embryonic bone formation during postnatal growth and in fracture repair. Mech Dev. 1998;71(1-2):65-76.

13. Jaenisch R, Bird A. Epigenetic regulation of gene expression: how the genome integrates intrinsic and environmental signals. Nat Genet. 2003;33(suppl):245-254.

14. Del Real A, et al. Differential analysis of genome-wide methylation and gene expression in mesenchymal stem cells of patients with fractures and osteoarthritis. Epigenetics. 2017;12(2):113-122.

15. Ghayor C, Weber FE. Epigenetic regulation of bone remodeling and its impacts in osteoporosis. Int J Mol Sci. 2016;17(9):E1446.

16. Smith ZD, Meissner A. DNA methylation: roles in mammalian development. Nat Rev Genet. 2013;14(3):204-220.

17. Challen GA, et al. Dnmt3a is essential for hematopoietic stem cell differentiation. Nat Genet. 2011;44(1):23-31.

18. Challen GA, et al. Dnmt3a and Dnmt3b have overlapping and distinct functions in hematopoietic stem cells. Cell Stem Cell. 2014;15(3):350-364.

19. Nishikawa K, et al. DNA methyltransferase 3a regulates osteoclast differentiation by coupling to an S-adenosylmethionine-producing metabolic pathway. Nat Med. 2015;21(3):281-287.

20. Wang C, Abu-Amer Y, O'Keefe RJ, Shen J. Loss of Dnmt3b in chondrocytes leads to delayed endochondral ossification and fracture repair. J Bone Miner Res. 2018;33(2):283-297.

21. Marsell R, Einhorn TA. The biology of fracture healing. Injury. 2011;42(6):551-555.

22. Schindeler A, McDonald MM, Bokko P, Little DG. Bone remodeling during fracture repair: The cellular picture. Semin Cell Dev Biol. 2008;19(5):459-466. 
23. Hilton MJ, et al. Notch signaling maintains bone marrow mesenchymal progenitors by suppressing osteoblast differentiation. Nat Med. 2008;14(3):306-314.

24. Kohn A, et al. Notch signaling controls chondrocyte hypertrophy via indirect regulation of Sox9. Bone Res. 2015;3:15021.

25. Dong Y, et al. RBPjkappa-dependent Notch signaling regulates mesenchymal progenitor cell proliferation and differentiation during skeletal development. Development. 2010;137(9):1461-1471.

26. Shang X, Luo Z, Wang X, Jaeblon T, Marymont JV, Dong Y. Deletion of RBPJK in mesenchymal stem cells enhances osteogenic activity by up-regulation of BMP signaling. PLoS One. 2015;10(8):e135971.

27. Kohn A, et al. Cartilage-specific RBPjк-dependent and -independent Notch signals regulate cartilage and bone development. Development. 2012;139(6):1198-1212.

28. Wang C, et al. Transient $\gamma$-secretase inhibition accelerates and enhances fracture repair likely via Notch signaling modulation. Bone. 2015;73:77-89.

29. Hadjiargyrou M, O'Keefe RJ. The convergence of fracture repair and stem cells: interplay of genes, aging, environmental factors and disease. J Bone Miner Res. 2014;29(11):2307-2322.

30. Frank O, et al. Real-time quantitative RT-PCR analysis of human bone marrow stromal cells during osteogenic differentiation in vitro. J Cell Biochem. 2002;85(4):737-746.

31. Dessau W, von der Mark H, von der Mark K, Fischer S. Changes in the patterns of collagens and fibronectin during limb-bud chondrogenesis. J Embryol Exp Morphol. 1980;57:51-60.

32. Stower H. Stem cells: Signalling to DNA methylation. Nat Rev Genet. 2013;14(9):595.

33. Bird A. DNA methylation patterns and epigenetic memory. Genes Dev. 2002;16(1):6-21.

34. Kang SH, et al. Transcriptional repression of the transforming growth factor- $\beta$ type I receptor gene by DNA methylation results in the development of TGF- $\beta$ resistance in human gastric cancer. Oncogene. 1999;18(51):7280-7286.

35. Serman L, Nikuseva Martic T, Serman A, Vranic S. Epigenetic alterations of the Wnt signaling pathway in cancer: a mini review. Bosn J Basic Med Sci. 2014;14(4):191-194.

36. Luo Y, et al. Differences in DNA methylation signatures reveal multiple pathways of progression from adenoma to colorectal cancer. Gastroenterology. 2014;147(2):418-29.e8

37. Joubert BR, et al. Maternal plasma folate impacts differential DNA methylation in an epigenome-wide meta-analysis of newborns. Nat Commun. 2016;7:10577.

38. Papathanasiou I, Kostopoulou F, Malizos KN, Tsezou A. DNA methylation regulates sclerostin (SOST) expression in osteoarthritic chondrocytes by bone morphogenetic protein 2 (BMP-2) induced changes in Smads binding affinity to the CpG region of SOST promoter. Arthritis Res Ther. 2015;17:160.

39. Lee J, et al. Epigenetic-mediated dysfunction of the bone morphogenetic protein pathway inhibits differentiation of glioblastoma-initiating cells. Cancer Cell. 2008;13(1):69-80.

40. Baubec T, et al. Genomic profiling of DNA methyltransferases reveals a role for DNMT3B in genic methylation. Nature. 2015;520(7546):243-247.

41. Cao Y, Yang H, Jin L, Du J, Fan Z. Genome-wide DNA methylation analysis during osteogenic differentiation of human bone marrow mesenchymal stem cells. Stem Cells Int. 2018;2018:8238496.

42. Yu F, Shen H, Deng HW. Systemic analysis of osteoblast-specific DNA methylation marks reveals novel epigenetic basis of osteoblast differentiation. Bone Rep. 2017;6:109-119.

43. Herlofsen SR, et al. Genome-wide map of quantified epigenetic changes during in vitro chondrogenic differentiation of primary human mesenchymal stem cells. BMC Genomics. 2013;14:105.

44. Purow B. Notch inhibition as a promising new approach to cancer therapy. Adv Exp Med Biol. 2012;727:305-319.

45. Ryeom SW. The cautionary tale of side effects of chronic Notch1 inhibition. J Clin Invest. 2011;121(2):508-509.

46. Rizzo P, et al. The role of notch in the cardiovascular system: potential adverse effects of investigational notch inhibitors. Front Oncol. 2014;4:384.

47. Baht GS, Vi L, Alman BA. The role of the immune cells in fracture healing. Curr Osteoporos Rep. 2018;16(2):138-145.

48. Dovey HF, et al. Functional $\gamma$-secretase inhibitors reduce $\beta$-amyloid peptide levels in brain. J Neurochem. 2001;76(1):173-181.

49. Tu X, et al. Physiological notch signaling maintains bone homeostasis via RBPjk and Hey upstream of NFATc1. PLoS Genet 2012;8(3):e1002577.

50. Das AT, Tenenbaum L, Berkhout B. Tet-On systems for doxycycline-inducible gene expression. Curr Gene Ther. 2016;16(3):156-167.

51. Ensrud KE. Epidemiology of fracture risk with advancing age. J Gerontol A Biol Sci Med Sci. 2013;68(10):1236-1242.

52. Clark D, Nakamura M, Miclau T, Marcucio R. Effects of aging on fracture healing. Curr Osteoporos Rep. 2017;15(6):601-608

53. Gerstenfeld LC, et al. Impaired fracture healing in the absence of TNF- $\alpha$ signaling: the role of TNF- $\alpha$ in endochondral cartilage resorption. J Bone Miner Res. 2003;18(9):1584-1592.

54. McKibbin B. The biology of fracture healing in long bones. J Bone Joint Surg Br. 1978;60-B(2):150-162.

55. Kolar P, et al. The early fracture hematoma and its potential role in fracture healing. Tissue Eng Part B Rev. 2010;16(4):427-434

56. Chung R, Cool JC, Scherer MA, Foster BK, Xian CJ. Roles of neutrophil-mediated inflammatory response in the bony repair of injured growth plate cartilage in young rats. J Leukoc Biol. 2006;80(6):1272-1280.

57. Andrew JG, Andrew SM, Freemont AJ, Marsh DR. Inflammatory cells in normal human fracture healing. Acta Orthop Scand. 1994;65(4):462-466.

58. Schmidt-Bleek K, et al. Inflammatory phase of bone healing initiates the regenerative healing cascade. Cell Tissue Res. 2012;347(3):567-573.

59. Okano M, Bell DW, Haber DA, Li E. DNA methyltransferases Dnmt3a and Dnmt3b are essential for de novo methylation and mammalian development. Cell. 1999;99(3):247-257.

60. Logan M, Martin JF, Nagy A, Lobe C, Olson EN, Tabin CJ. Expression of Cre Recombinase in the developing mouse limb bud driven by a Prxl enhancer. Genesis. 2002;33(2):77-80.

61. Reynolds DG, et al. muCT-based measurement of cortical bone graft-to-host union. J Bone Miner Res. 2009;24(5):899-907. 